\title{
FACILE GENERATION OF BIOMIMETIC SUPPORTED LIPID BILAYERS ON CONDUCTING POLYMER SURFACES FOR MEMBRANE BIOSENSING
}

\author{
A Thesis \\ Presented to the Faculty of the Graduate School \\ of Cornell University \\ In Partial Fulfillment of the Requirements for the Degree Of \\ Master of Science
}

by

Hui Su

[December 2019] 
(C) 2019 Hui Su 


\begin{abstract}
Membrane biosensors that can rapidly sense pathogen interaction and disrupting agents are needed to identify and screen new drugs to combat antibiotic resistance. Bioelectronic devices have the capability to read out both ionic and electrical signals, but their compatibility with biological membranes is somewhat limited. Supported lipid bilayers (SLBs) have served as useful biomimetics for myriad research topics involving biological membranes. However, SLBs are traditionally made on inert, rigid, inorganic surfaces. Here, we demonstrate a versatile and facile method for generating SLBs on a conducting polymer surface in a bioelectronic device using a solvent assisted lipid bilayer (SALB) technique. We use this bioelectronic device to form mammalian and bacterial membrane mimetics to sense the membrane interactions with a bacteria toxin and an antibiotic compound, respectively. The SALB formation method is compatible with many membrane compositions that will not form via common vesicle fusion methods and works well in microfluidic devices. These features, combined with the massive parallelization possible for lithography, can lead to miniaturized multiplexed devices for rapid data acquisition necessary to identify antibiotic targets that specifically disrupt bacterial membranes and not mammalian. On the other hand, devices that can quickly screen bacteria toxin interactions with mammalian cell surfaces will be useful for identifying bacteria friends or foes as antibiotic resistance grows and our desire to classify the microbiomes from various sources increases.
\end{abstract}




\section{BIOGRAPHICAL SKETCH}

Hui $\mathrm{Su}$ received a B.S degree in chemical and biomolecular engineering with a biomanufacturing minor at North Carolina State University in 2017. Following her undergraduate studies, she further pursued a M.S degree in chemical and biomolecular engineering at Cornell University in Dr. Susan Daniel's research group in October 2017. 


\section{ACKNOWLEDGEMENTS}

I would like to thank many people who have helped and encouraged me to finish my master thesis at Cornell University. First, I must express gratitude to my advisor, Dr. Susan Daniel, as a great mentor for my research. She has always been encouraging and supportive for my ideas and guided me to find the right path to my project. She also provided me with the best opportunity to work with excellent collaborators. Thanks to her great dedication in helping me edit the manuscript, I have a paper submitted just before my graduation. I couldn't have possibly achieved this accomplishment without her steady support.

I would also like to thank our collaborators Dr. Róisín Owens, Dr. Anna-Maria Pappa, and Dr. Sahika Inal for their contributions on the electrochemical measurement and modeling as a crucial component of my final thesis. Not only did they perform the analysis that helped me complete my project, their dedication to science also inspired me as a researcher and encouraged me to explore the field more in the future.

I must extend my enthusiastic thanks to my committee member, Dr. Christopher Ober, and his student, Hai Tran, for giving me a lot of insights about the polymers and helping me open up another horizon.

Finally, I owe gratitude to all the students in Professor Susan Daniel's research group. Though I can't possibly mention everybody here, I still want to thank Han-Yuan Liu for his advice for my experiment and the revision of the manuscript. I would like to thank everybody for such a great lab environment and how helpful they have been for my research. 


\section{TABLE OF CONTENTS}

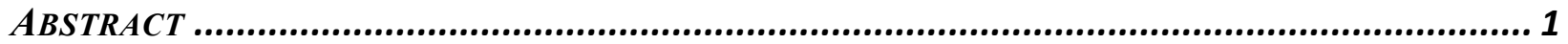

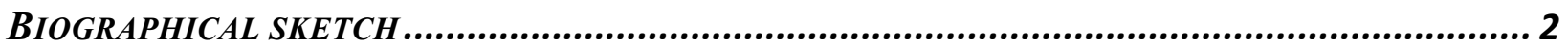

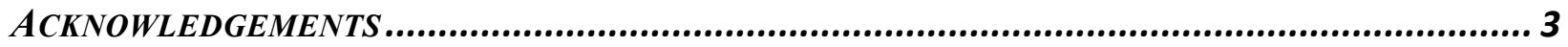

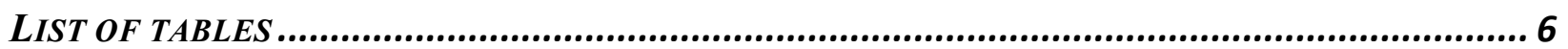

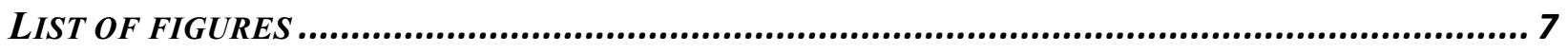

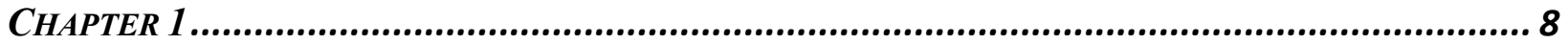

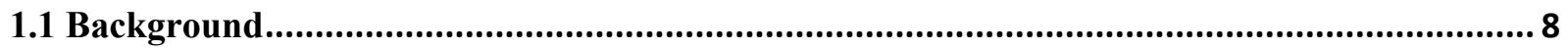

1.2 Conducting Polymer (CP) and Organic Electrochemical Transistor (OECT) ...................... 10

1.3 Solvent Assisted Lipid Bilayer (SALB) and its Electrical Properties ................................... 12

1.4 Electrical Measurement of Lipid Bilayers by Electrochemical Impendence Spectroscopy

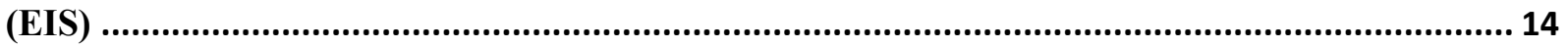

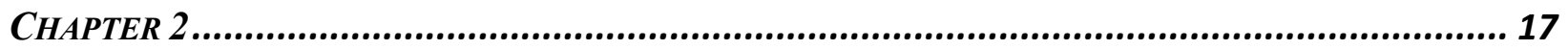

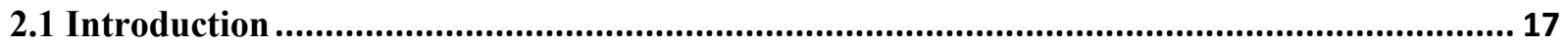

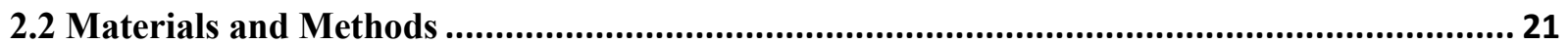

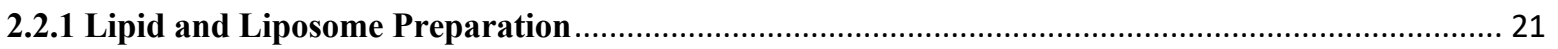

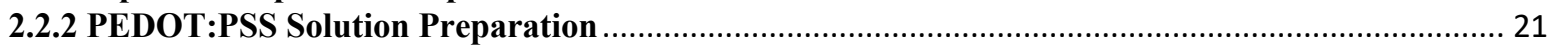

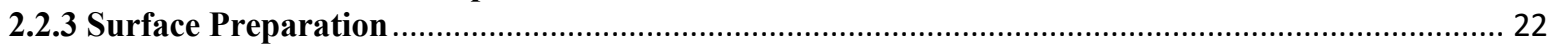

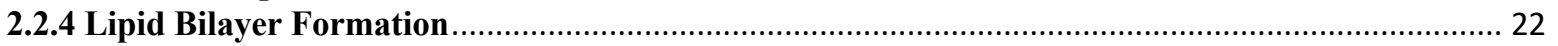

2.2.5 Fluorescence Recovery after Photobleaching (FRAP) on Bilayers .............................................. 23

2.2.6 Quartz Crystal Microbalance with Dissipation (QCM-D) Monitoring Lipid Bilayer Formation... 23

2.2.7 Quartz Crystal Microbalance with Dissipation (QCM-D) Monitoring the Insertion of Alpha-

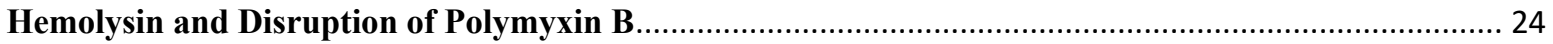

2.2.8 Electrochemical Impedance Spectroscopy Measurement for Lipid bilayer formation and Detection

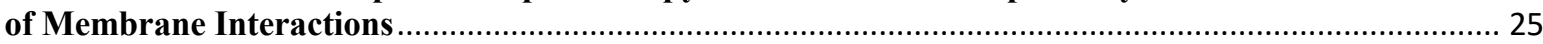

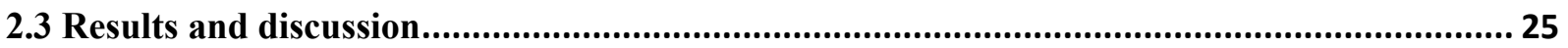

2.3.1 Supported Lipid Bilayer Formation by Vesicle Fusion or Solvent-Assisted Methods .................... 25

2.3.2 Characterization of Lipid Bilayer Planarity and Two-dimensional Fluidity on Glass and

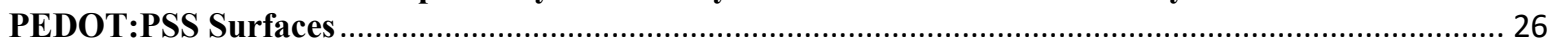

2.3.2 Monitoring of the Real-Time Lipid Bilayer Formation on $\mathrm{SiO}_{2}$ and PEDOT:PSS Films by SA

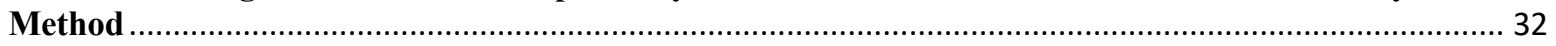

2.3.3 Characterization of Electrical Properties of Lipid Bilayers on PEDOT:PSS Electrode Surfaces .. 38

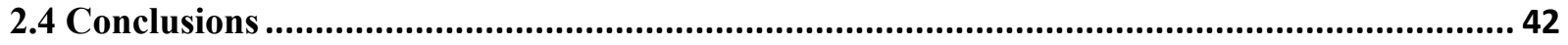

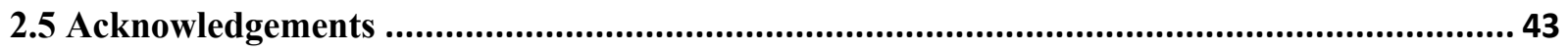

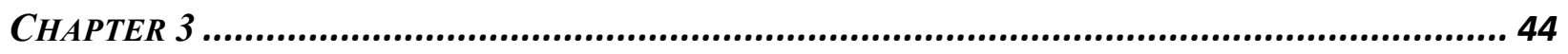

3.1 SALB is the Platform to Study Variety of Biomembrane Activities ...................................... 44

3.1.1 Investigation of the Lipid and Solvent Interaction with PEDOT:PSS Film.................................. 44 
3.1.2 The Interaction Between Lipid Bilayer and Bacterial Toxin and Antibacterial Drug

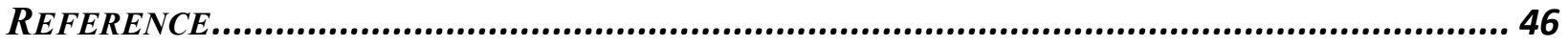

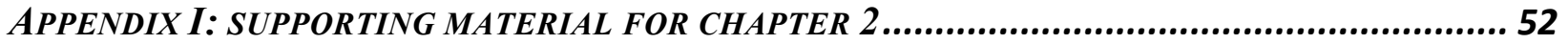

S1. Vesicle Fusion on PEDOT:PSS Film or Bare Sensor Surface Measured by QCM-D ........... 52

S2. EIS Modelling of the Mammalian and the Bacterial Membranes ................................... 54

S3. The Solvent Exchange Control Measured by QCM-D ............................................... 55

S4. The Insets of the Final Solvent Exchange of QCM-D Monitoring the SALB on Glass and

PEDOT:PSS Surfaces...................................................................................... 56 


\section{LIST OF TABLES}

Chapter 2

Table 1 - Diffusivity and Mobile Fraction of Mammalian Membranes on Glass and PEDOT:PSS using SA and VF

Table 2 - Diffusivity and Mobile Fraction of Bacterial Membranes on Glass and PEDOT:PSS using SA and VF

Table 3 - Quantitative analysis of mammalian and bacteria model membrane on $\mathrm{SiO} 2$ surfaces and PEDOT:PSS films 


\section{LIST OF FIGURES}

Chapter 1

Figure 1 - The molecular structure of PEDOT:PSS and basic schematic structure of OECTs

Figure 2 - Phospholipids lyotropic phases in different organic solvent contents.

Figure 3-The basic schematic setup for electrochemical impedance spectroscopy (EIS) measurement

\section{Chapter 2}

Figure 1 - The microfluidic channel setup for the formation of the SALBs and setup for EIS measurement for mammalian and bacterial membrane disruption

Figure 2 - FRAP characterization of mammalian membrane by VF method and SA method

Figure 3 - FRAP characterization of bacterial membrane by VF method and SA method Figure 4 - QCM-D monitoring the mammalian and bacterial membranes by SA method Figure 5 - QCM-D monitoring the SALB interaction with $\alpha$-HL or PMB on PEDOT:PSS films

Figure 6 - EIS impedance monitoring of the SALBs on Au/PEDOT:PSS surfaces 


\section{CHAPTER 1 \\ INTRODUCTION}

\subsection{Background}

The cell membrane is a biological membrane that separates cytoplasm from the extracellular environment, which is vital for signaling, transporting, and compartmentalization of reactions. The cell membrane structure is made of a double-layer of lipid molecules that oppose each other due to their amphiphilic chemical nature composed of a hydrophilic phosphate head group and hydrophobic fatty acid chains. Phospholipids, glycolipids, and sterols are three classes of lipids in biological membranes, though there is great diversity of lipids within these classes. The compositions of different lipids vary in organelles of the cell all the way up to specificity in organisms. In addition to lipids, sugars and membrane proteins insert into membranes, which are also crucial for cell functioning. Imagining how these lipids and proteins might arrange in the membrane structure lead to Singer and Nicholson developing what is called, "the fluid mosaic model" in 1972, where they proposed the fundamental structure of the lipid molecules and membrane proteins within it as a "brick and mortar" arrangement. ${ }^{1}$ Lipid membranes create the environment for membrane proteins to support structure and function, and thus serve as the mortar in this model, while the bricks are the proteins. This model, though quite useful, was rather simple in design, proposing that all species were full mobile and that the membrane was a lot more dilute that what we think of today. Nowadays, biological membranes are exhaustedly studied to understand functions of the lipids and protein, including their critical protein-lipid interactions, protein recognition, and signal transduction..$^{2-4}$

A useful tool for such studies emerged as the supported lipid bilayer (SLB), demonstrated by Tamm and McConnell in $1985 .{ }^{5}$ SLBs have been used as platforms to study membrane 
processes, such as membrane domain fusion, membrane disruption with antimicrobial, and viral fusion kinetics. ${ }^{6-8}$ SLBs can be fabricated by using Langmuir-Blodgett deposition, lipid-detergent micelles, and vesicle fusion. ${ }^{9-11}$ Vesicle fusion (VF) is the most conventional method to form SLBs due to the liposome self-assemble into SLBs characteristic onto the solid supports and the potential that preserves the natural structure of biological membranes. Besides the pure phospholipid bilayers, our group has demonstrated how to fabricate SLBs from native cell membrane components and rupture with fusogenic vesicles. ${ }^{8}{ }^{12}$ In order to observe vesicle fusion and rupture to form SLBs, two essential factors have to be fulfilled: adequate adhesion energy, which means vesicles adsorb and stabilized onto the surface; sufficient coverage, which denotes vesicles reach critical coverage and start rupture to fuse into a continuous lipid bilayer. ${ }^{13}$

In some cases, forming lipid bilayers by using VF is not straightforward, some conditions need to be adjusted. To increase the adhesion energy, vesicle composition can be changed to increase the electrostatic interactions between vesicles and substrates. Moreover, the pre-coated substrate with the polymer can also enhance the electrostatic interactions. ${ }^{14}$ Another strategy to add divalent cations, such as $\mathrm{Ca}^{2+}$ and $\mathrm{Mg}^{2+}$, which are considered as fusion promoters, to elevate and adsorption and rupture of the vesicles and lipid bilayer formations. ${ }^{15}$

Self-assembled SLBs are stable and robust two-dimensional fluid surroundings, allow lipids and membrane proteins diffuse laterally. Such features mimic well the cell membrane features for analyzing membrane processes, especially because the planar structure is compatible with a variety of surface analytical tools, such as atomic force microscopy (AFM) $)^{13}$, total internal reflection microscopy (TIRFM) ${ }^{16}$, and quartz crystal microbalance with dissipation (QCM-D) ${ }^{17}$.

Although SLBs has tremendous merits, it has not had as much impact in the rising fields of biosensing and bioelectronics due to the restrictions of the solid substrates. SLBs prefer to form 
on hydrophilic and smooth silica-based inorganic materials, such as glass and mica. Such materials are generally inert so that it is impossible for ion and electrons to transduce for bioelectronics and biosensing purposes. Therefore, integrating in a biocompatible conducting polymer (CP) as the biological interfacial between SLBs and electronic material is critical to opening these applications.

In this chapter, background information about the typical material and device used for electrochemical measurement is provided. The challenges between of bioelectronics and biological worlds, especially using SLBs as a platform for drug screening will be discussed. These barriers motivated this project to develop biomembrane mimetics compatible with $\mathrm{CP}$ surfaces and further characterize the membrane properties by using optical and electrochemical measurements.

\subsection{Conducting Polymer (CP) and Organic Electrochemical Transistor}

\section{(OECT)}

Poly(3,4-ehtylenedioxythiophene), which is the p-type hole conducting material, doped with poly(styrene sulfonate) (PEDOT:PSS), is electrically conductive due to the delocalized $\pi$ electrons in the chemical structure (Fig. 1A). ${ }^{18}{ }^{19}$ PEDOT:PSS has outstanding hole conductivity, ${ }^{19}$, ${ }^{20}$ stability in aqueous electrolyte, ${ }^{21}$ soft mechanical properties, ${ }^{22}$ and high transparency, ${ }^{18}$ which make PEDOT:PSS a superb material as a biological interface material in between electronic material and biological environment for both electrochemical measurement and optical microscopy. Specific to the surface morphology, PEDOT:PSS changes from coiled gel particles to the pancake-like structure after spin coating on the surface (Fig. 1B). ${ }^{19,23}$ After the polymer film swells in the aqueous environment, the loose structure of the film can aid the ion transport. ${ }^{20}$ The co-solvent additive, ethylene glycol (EG), is commonly used to modify the film structure as EG

increases the PEDOT:PSS domains and crystallinity which increase the hole conductivity. ${ }^{19,} 20$ Moreover, 3-glycidyloxypropyltrimethoxysilane (GOPS) is a crosslinker to enhance the polymer 
film stability and reduce the delamination. ${ }^{24}, 25$ Although GOPS causes the reduction of conductivity, it is still sufficient for electrochemical measurements. ${ }^{24,26}$

With the balanced features of merit, PEDOT:PSS has been considered as the champion material for the CP layer in organic electrochemical transistors (OECTs). OECTs has a gate electrode, a metallic source, and a drain (Fig. 1C). Since PEDOT:PSS is the p-type semiconductor, OECTs operate in the depletion mode. When gate voltage $\left(\mathrm{V}_{\mathrm{G}}\right)$ is zero, OECT is "ON" due to the holes current in the channel. When positive gate voltage applies to the electrolyte, it pushes cations to penetrate into the channel. The cations can form ionic bonds with PSS; therefore, the holes in the PEDOT backbone are no longer stable and flow out through the drain. As a result, the current decreases, and OECT reaches "OFF" mode. ${ }^{25,27}$ Previous work indicates OECTs have extremely high sensitivity due to the absence of insulator between channel and electrode. ${ }^{25}, 28$ Also, it facilitates ions and electrons to enter the material, which amplifies the signal-to-noise ratio. ${ }^{24,25,28}$ Moreover, for biological applications, the electrolyte typically is the buffer, which better mimics the biological environment.

(A)

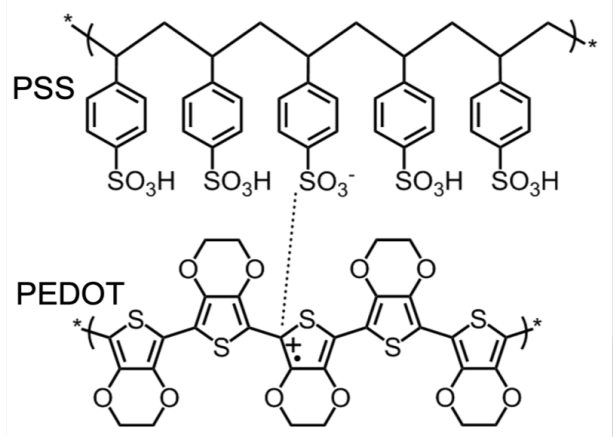

(B)

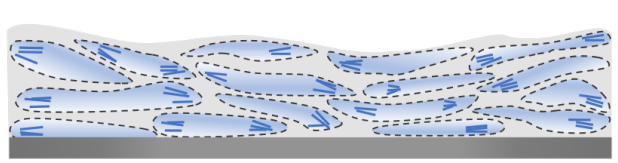

(C)

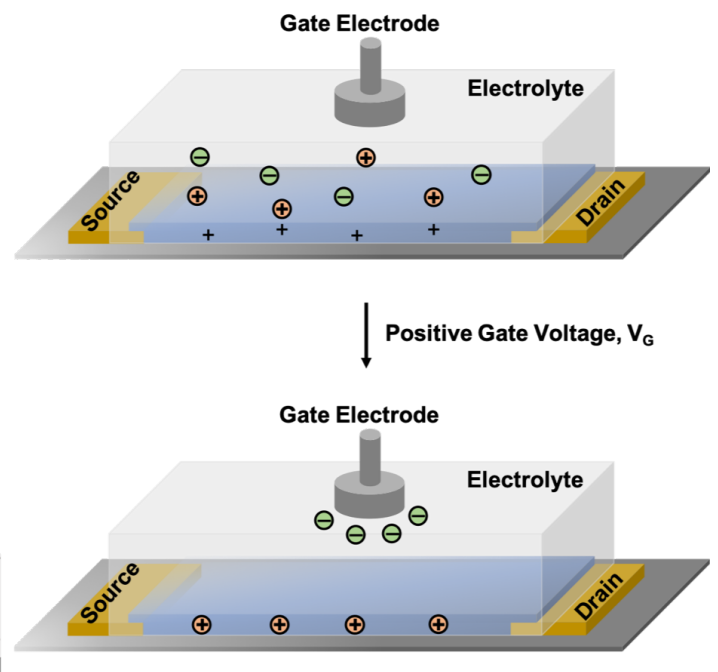

Figure 1. (A) The molecular structure of PEDOT:PSS. The dash line indicates the ionic bond forming between PSS and PEDOT. (B) The morphology of PEDOT:PSS with solvent additive ethylene glycol (EG) after coating on the 
glass substrate (dark grey). EG alter the structure of PEDOT:PSS, and it forms PSS rich regions (light grey), PEDOT:PSS rich regions (blue), and crystalline domains (blue lines). (C) The typical structure of an organic electrochemical transistor (OECT). The substrate is glass (dark grey), and the yellow components indicate source (left) and drain (right). PEDOT:PSS (blue) is coated in the channel. The top part is the gate electrode (grey), which contacts with the electrolyte. The figure illustrates the depletion mode of OECT. After the gate electrode applies positive gate voltage $\left(V_{G}\right)$, it pushes cations from electrolyte into the PEDOT:PSS films, and holes flow out through the drain.

Thus, OECTs have revealed promising results in real-time monitoring of interactions between membrane mimetics and antimicrobial compounds. ${ }^{28,}{ }^{29}$ Since the lipid membrane is highly electrically resistant, behaving like an insulator, it blocks the ion transport in between the electrolyte and the semiconductor channel. ${ }^{28,30}$ Once adding an antibacterial compound to disrupt the lipid monolayers (MLs), the detergent-like mechanism will pull apart the membrane, which results in "leaks" in the membrane. Therefore, the ions can penetrate into the channel to increase the conductance. ${ }^{28}$ Another work successfully demonstrates the insertion of transmembrane protein (ion channel) into the lipid bilayer, which results in the increase of ion transport across the membrane and the conductance changes accordingly. ${ }^{29}$ However, such experiments utilize MLs or very limited compositions of lipids due to the difficulties of self-assembling SLBs on PEDOT:PSS. Therefore, finding an alternative method, which provides high quality of the SLBs and tunable lipid compositions, is essential to further extend OECTs applications into the biosensing fields.

\subsection{Solvent Assisted Lipid Bilayer (SALB) and its Electrical Properties}

Common VF methods prefer hydrophilic and smooth surfaces, but PEDOT:PSS swells in the buffer, which makes the surface roughness increase and unfavor spontaneous vesicles rupture onto the polymer film. ${ }^{31} \mathrm{VF}$ requires large adhesion force between the surface and vesicles that can trigger vesicle rupture and spread spontaneously, but negatively charged polymer - PSS- leads to vesicles with some negative charges vesicles harder to overcome the electrostatic forces such that the Van der Waals forces can promote rupture and form the lipid bilayers. ${ }^{32}$ Therefore, forming 
SLBs on the PEDOT:PSS can be a challenge, especially for biomembranes that attempt to capture the typical negative surface charges of native membranes, such as found in bacterial membranes.

Solvent assisted lipid bilayer (SALB) employs the concept of phospholipids lyotropic phase changes under different organic solvent conditions. As the buffer (water) content increases from a primarily organic phase initially, phospholipids undergo a series of phase transitions, from inverted micelles, lipid molecules, lipid micelles, and vesicles in the bulk; meanwhile, lipid micelles deposited onto the bulk-polymer interface spontaneously assemble into a contiguous lipid bilayer (Fig. 2A-D). Solvent-assist (SA) method bypasses the vesicle preparation steps, and adhesion and rupture processes necessary in VF method. More importantly, this method does not require high adhesion energy as VF method does for vesicle rupture and to be able to spread spontaneously on the surface. Although the exact mechanism still remains to be elucidated, SALBs demonstrate robust and reproducible qualities on new types of surfaces that are intractable by the common VF method, such as nanoporous gold and aluminum oxide. ${ }^{33}, 34$ Additionally, SA method can extend the lipid compositions that are difficult for the common VF method, for example high cholesterol content lipid bilayers and negatively charged bilayers. ${ }^{35,36}$ Due to the benefits of SALBs, there is great potential open for the SALB to be used in a variety of biological applications. 
(A)

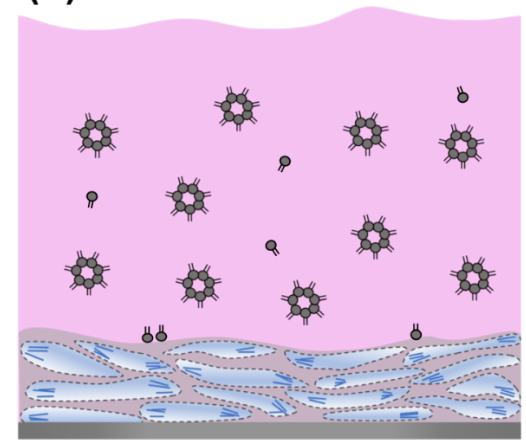

(C)

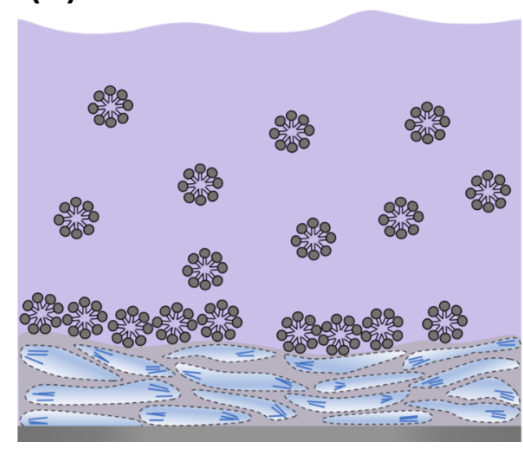

(B)

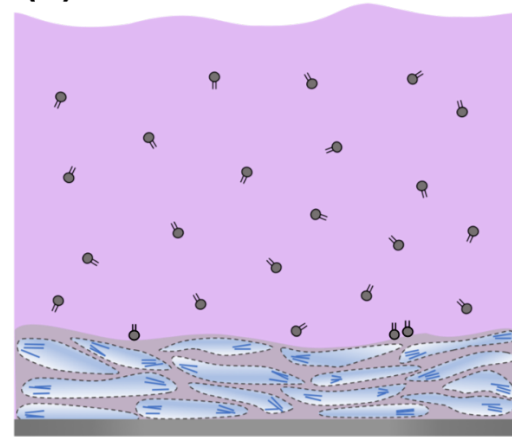

(D)

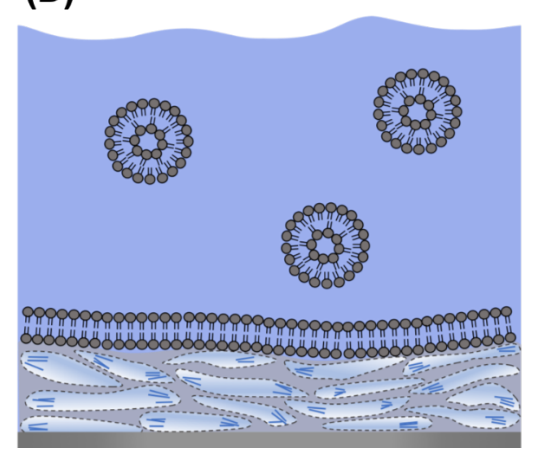

$100 \%$ organic solvent

Bulk Interface

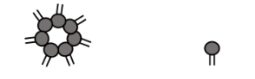

Inverted Lipid

micelles molecules

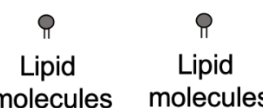

molecules

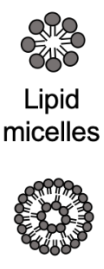

Vesicles

$100 \% \mathrm{NaCl} / \mathrm{Tris}$ Buffer

Figure 2. Phospholipids lyotropic phases in different organic solvent contents. (A) In organic solvent, the dominant phase in the organic solvent is inverted micelles, and few lipid molecules also exist. (B) With the increase of buffer content, inverted micelles disassemble into phospholipid molecules. (C) Continuously increase of the buffer fraction, lipid molecules reassemble into normal lipid micelles, and lipid micelles deposit onto the surface. (D) Upon the buffer exchange with the majority of the organic solvent, lipid micelles quickly assemble into a contiguous lipid bilayer on the buffer-surface interface, and vesicles are formed in the bulk.

\subsection{Electrical Measurement of Lipid Bilayers by Electrochemical Impendence}

\section{Spectroscopy (EIS)}

Electrochemical impedance spectroscopy (EIS) is an important approach to study membrane electrical properties. The real-time changes of resistance and capacitance elucidate the membrane activity, such as membrane stability, membrane permeability, and membrane interactions. By characterizing the impedance spectra before bilayer formation and after bilayer formation on PEDOT:PSS coated electrodes, an electrical equivalent circuit, which consists of a series of connected impedance elements, can be modeled and analyzed for membrane resistance and capacitance (Fig. 3). Since the lipid bilayers are electrically insulating, the contiguous 
membranes have low ion transduction and high electrical resistance. ${ }^{30,37,38}$ This feature is critical for monitoring ion transport across the membrane. In VF method, it is challenge to form lipid bilayers on the PEDOT:PSS. Even with the formation of bilayers, the membrane resistance is still insufficient for ion transport measurement. ${ }^{29}, 39,40$ However, SA method provides a better alternative to forming a highly sealed lipid bilayer that can be used for potential ion transporting or membrane interaction characterizations. ${ }^{37}$

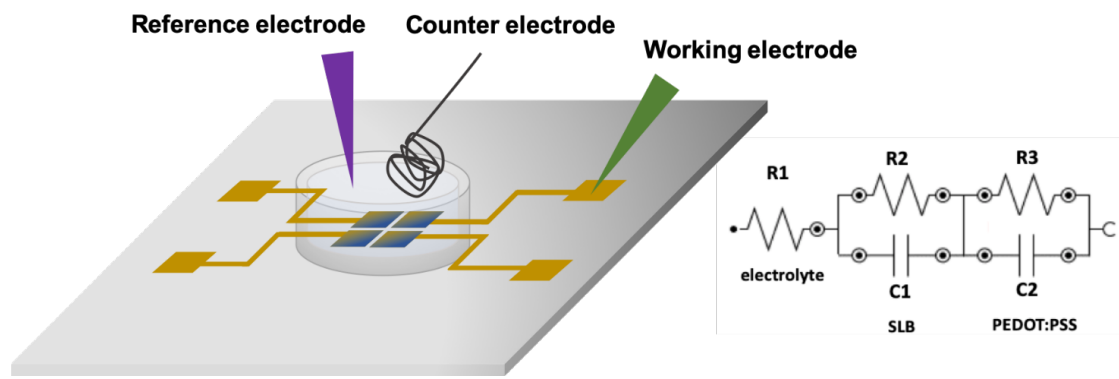

Figure 3. The basic schematic setup for electrochemical impedance spectroscopy (EIS) measurement (left). A cured polydimethylsiloxane (PDMS) well sticks to the glass chip (light grey). The PDMS well contains the electrolyte for the measurement (hollow cylinder). The conventional implementation consists of a reference electrode, a platinum mesh counter electrode, and a gold coated working electrode. The system equivalent electrical circuit (right) includes a resistance for electrolyte (R1), followed by two RC parallel impedance: impedance of the SLB, which consists a resistor (R2) in parallel with a capacitor (C1); impedance of PEDOT:PSS, which consists of a resistor (R3) in parallel with a capacitor $(\mathrm{C} 2)$.

In this chapter, the merits of SALB are discussed. This method bypasses the conventional liposomes preparation steps and rupturing process. Moreover, the requirement of adhesion energy is significantly lower than the VF method. Thus, this method is capable of self-assembled on the conducting polymer, which is a challenge for the VF method. Fabricating lipid bilayers on the PEDOT:PSS film and investigating membrane properties by EIS measurement have the competent and potential for studying the membrane properties and membrane biosensing.

In the next chapter, two SALB models are demonstrated for membrane fabrication and biosensing applications on the conducting polymer. To be specific, a mammalian membrane model 
and a bacterial membrane model are built on the PEDOT:PSS film by using the SALB method, and later use for monitoring the membrane interaction with a bacterial toxin and an antibiotic drug, respectively. This method is a feasible approach to form lipid bilayers, and the quality is comparable to the lipid bilayers that form by the conventional VF method. More importantly, this method can form highly sealed membranes that are suitable for membrane biosensing applications. 


\section{CHAPTER 2 \\ FACILE GENERATION OF BIOMIMETIC SUPPORTED LIPID BILAYERS ON CONDUCTING POLYMER SURFACES FOR MEMBRANE BIOSENSING ${ }^{1}$}

\subsection{Introduction}

Biological membranes comprised of phospholipids, sterols, and proteins define the inside of the cell from the outside and regulate the critical cellular functions at this interface. Such critical functions include transporting material across the membrane ${ }^{41}$, transducing signals through $i t^{42}$, and recognizing events at that interface ${ }^{2}$. The lipid bilayer is the essential structure of biological membranes and widely mimicked as a model system to investigate interfacial phenomena between the cell surface and external compounds such as membrane targeting antibiotics ${ }^{43}$, host-pathogen interactions ${ }^{6}$, and ion or hormone flux through membrane protein channels ${ }^{44}$. Supported lipid bilayers (SLB) have emerged as a prominent platform used in many of these investigations. An essential feature of SLBs is their planar geometry, making them compatible with many surfacebased analytical tools and optical microscopy. Since first demonstrated by Tamm and McConnell in $1985,{ }^{5}$ most SLBs are formed on transparent, hydrophilic, inorganic surfaces like glass or silica, because of the ease of SLB self-assembly by vesicle fusion (VF). ${ }^{45}, 46$ This self-assembly feature lends itself well to coating the channels of glass and oxidized polydimethylsiloxane microfluidics with lipid bilayers, and with the exponential growth of microfluidic applications in the last two decades, SLBs have risen to prominence as an important tool for biologists and bioengineers investigating membrane phenomena; yet they are not without limitations.

\footnotetext{
${ }^{1}$ Su, H.; Liu, H.-Y.; Pappa, A.-M.; Hidalgo, T. C.; Cavassin, P.; Inal, S.; Owens, R. M.; Daniel, S., Facile Generation of Biomimetic-Supported Lipid Bilayers on Conducting Polymer Surfaces for Membrane Biosensing. ACS Applied Materials \& Interfaces 2019, 11 (47), 43799-43810.
} 
Although silica-based surfaces have served well for many applications, they are limiting for a variety of applications that rely on transport of information across the bilayer, such as in biosensing and signal transduction, ion flux changes, and membrane disruption. These applications are precluded from investigation by the electrically insulting and inflexible silica-based surfaces that can also denature proteins protruding underneath the bilayer. Opening the field to these more sophisticated studies requires new surfaces and tools that can overcome these limitations and enable new ways to interrogate the system, without losing the features that made silica-based surfaces a beneficial platform.

Organic materials, like polymers, are indeed a preferred means for connecting the biological with the physical worlds, owing to the ability to tune their mechanical properties to mimic those of native tissues, i.e., they can be compliant and generally inert. Towards this end, many researchers have incorporated polymers as thin coatings on silica surfaces as a means to overcome the deleterious effects on membrane proteins. ${ }^{47}$ Inert polymer cushions minimize the interaction of membrane proteins in the SLB with the underlying support and help to preserve the native protein function. ${ }^{47-49}$ However, moving towards polymers that are not just invisible to the biophysical phenomena under study, but offer capabilities to actively participate in the transduction of information, is the state-of-the-art. Indeed, conducting polymers (CPs) have lately received increasing interest for interfacing with SLBs as they can preserve protein function as a cushion, and simultaneously offer a direct means to sense and transduce signals across the membrane. Combining the inherent insulating properties of biomembranes with biocompatible CPs renders their arrangement on electrodes as a very attractive platform to measure membrane phenomena with a direct electrical readout. 
In a recent study, ${ }^{50}$ we have shown successful SLB formation by VF on top of CP surfaces made of poly(3,4-ethylenedioxythiophene) polystyrene sulfonate (PEDOT:PSS), a commonly used material for coupling with biological systems. ${ }^{25,51-53}$ Based on our observations, only certain vesicle compositions could rupture and self-assemble into SLBs on this CP. The optimized lipid composition was found to be a 7:3 ratio of 1,2-diphytanoyl-sn-glycero-3-phosphocholine (DPhPC) and 1,2-diphytanoyl-sn-glycero-3-phosphoethanolamine (DPhPE), made of lipids derived from archaea bacteria. Though these same lipids are often used in black lipid membrane experiments to great effect, ${ }^{54-56}$ our goal here was to expand this platform to create mammalian and bacterial mimetics that are more relevant to biosensing applications in host-pathogen sensing and in the development of new classes of antibacterial compounds specific for gram-negative species.

Solvent-assisted lipid bilayer formation (SALB) is an emerging method for forming many types of lipid bilayers on diverse substrates such as silicon dioxide, gold, aluminum oxide, and graphene that is impossible to achieve with VF. ${ }^{36,57-60}$ This method suspends lipid micelles in an isopropanol-water mixture that is exposed to the surface of interest. During a slow replacement with an aqueous buffer, adsorbed lipid material at the surface assembles into a planar, supported lipid bilayer (Fig. 1A) ${ }^{61}$ Importantly, unlike the stringent requirements of vesicle compositions and surface properties for bilayer formation by VF, ${ }^{62-64}$ SALB bypasses vesicle preparation and rupture, enabling lipid bilayer formation in non-ideal conditions for vesicle fusion. One situation where this is particularly relevant is in forming negatively-charged bacterial membranes, which otherwise would be repelled from a negatively charged surface like glass or PEDOT:PSS. ${ }^{21}$ Additionally, other lipids types with limited affinity for PEDOT:PSS, such as zwitterionic 1palmitoyl-2-oleoyl-glycero-3-phosphocholine (POPC) - a common constituent of mammalian membranes - are difficult to form into bilayers by VF, a result of reduced van der Waals forces to 
drive the vesicle rupture. We show here both types of lipid compositions readily form SLBs using the SALB method on PEDOT:PSS.

In this work, we illustrate the assembly of mammalian and bacterial model membranes on both glass slides and PEDOT:PSS thin films by the SALB method and contrast it with the VF method. We characterize the bilayer assembly and final mechanical properties with fluorescence recovery after photobleaching (FRAP) and quartz crystal microbalance with dissipation monitoring (QCM-D). Bilayer formation on PEDOT:PSS electrode surfaces was further validated by electrochemical impedance spectroscopy measurement (EIS). We demonstrate the electrical sensing capability of this platform with two examples (Fig. 1B). We measure the interactions between a mammalian model membrane and alpha-hemolysin $(\alpha-H L)$, a pore-forming toxin from Staphylococcus aureus, ${ }^{65}$ and antibiotic interactions between bacterial model membrane and Polymyxin B (PMB), a cationic peptide used to treat gram-negative bacterial infections. ${ }^{66}$
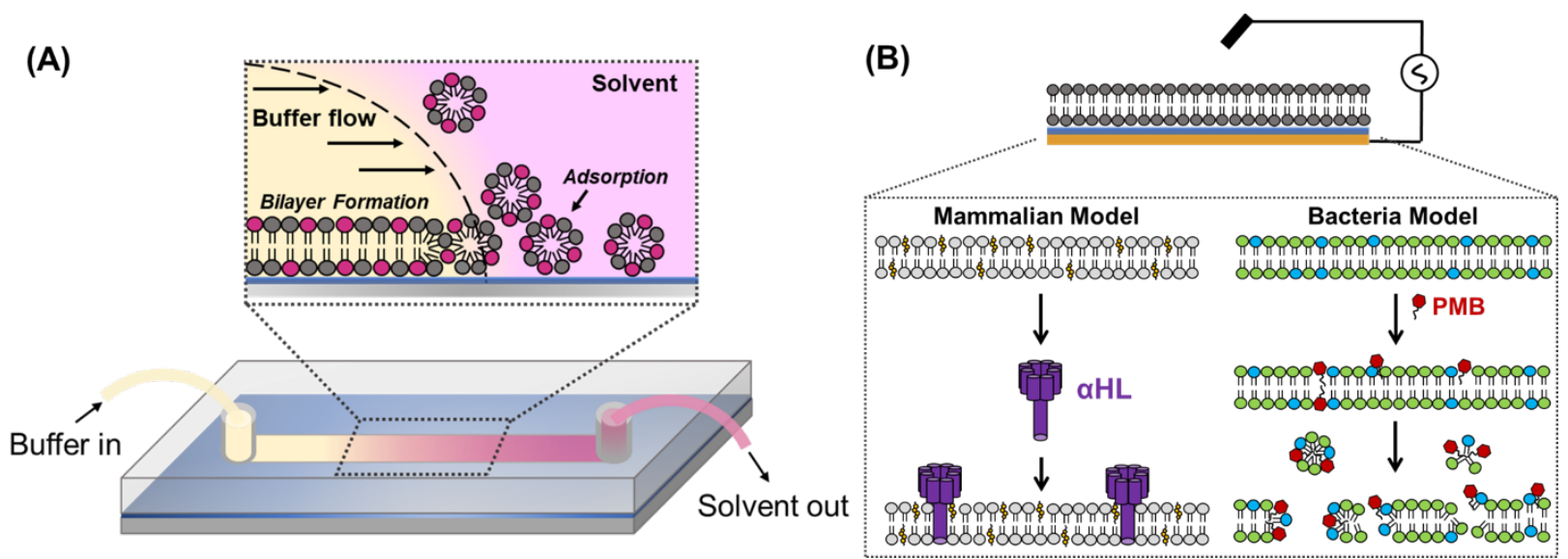

Figure 1. (A) Solvent assisted lipid bilayer formation (SALB) inside a microfluidic channel. The magenta color denotes the solvent phase containing lipid micelles doped with Texas red labeled lipids; the yellow phase represents the aqueous buffer flowing in to replace the solvent and forming the supported bilayer at the channel surface in the process. Texas red lipids are added for FRAP experiments described in the main text. (B) The EIS configuration used in this work. The thin blue layer represents the PEDOT:PSS film and the gold layer represents the gold contact supporting the polymer and planar bilayer. Below the diagram are cartoon representations of the two bilayers we formed with this method, and the toxin ( $\alpha$-hemolysin) or compound (Polymyxin B) used to disrupt them. POPC (light gray) and cholesterol (yellow) are principle components of the mammalian bilayer and POPE (green) and POPG (blue) are the components used for the bacterial bilayer. 


\subsection{Materials and Methods}

\subsubsection{Lipid and Liposome Preparation}

In this work, two membrane formulations were used: $80 \%(\mathrm{~mol} / \mathrm{mol}) 1$-palmitoyl-2-oleoylglycerol-3-phoshocholine (POPC) and 20\% cholesterol, which represents the principal components of a typical mammalian cell membrane; and $80 \%$ (mol/mol) 1-palmitoyl-2-oleoyl-snglycero-3-phosphoethanolamine (POPE) and 20\% 1-palmitoyl-2-oleoyl-sn-glycero-3-phospho(1'-rac-glycerol) (POPG), which represents typical components of a bacterial cell membrane. POPC, Cholesterol (ovine wool, >98\%), POPE, and POPG (sodium salt) were purchased from Avanti Polar Lipids (Alabaster, AL). Lipids were used as received.

For vesicle fusion (VF) experiments, liposomes were prepared by mixing lipids in chloroform at desired concentration. After mixing, the majority of chloroform was evaporated under a stream of ultra-pure nitrogen. Residual chloroform was completely removed by placing the mixture in a desiccator under vacuum for 3 hours. The lipid film was rehydrated with phosphate buffered saline (PBS), composed of $5 \mathrm{mM} \mathrm{NaH}_{2} \mathrm{PO}_{4}, 5 \mathrm{mM} \mathrm{Na}_{2} \mathrm{HPO}_{4}, 150 \mathrm{mM} \mathrm{NaCl}$ at $\mathrm{pH} 7.4$, to achieve the desired concentration. The solution was frozen and thawed once, and extruded 15 times through a $50 \mathrm{~nm}$ pore polycarbonate membrane (Whatman Nucleopore, GE Health).

For solvent assisted lipid bilayer (SALB) experiments, lipid mixtures were prepared by using the same procedure as liposomes; except instead of using PBS buffer to rehydrate the lipid films, a solution of $70 \% \mathrm{v} / \mathrm{v} 18.2 \mathrm{M} \Omega-\mathrm{cm}$ water (Siemens Purelab Ultra water purification system) and $30 \% \mathrm{v} / \mathrm{v}$ isopropanol (VWR) was substituted to obtain the desired solution concentration.

\subsubsection{PEDOT:PSS Solution Preparation}

95\% v/v Clevios ${ }^{\mathrm{TM}} \mathrm{PH} 1000$ (Heraeus), 5\% v/v ethylene glycol (Sigma-Aldrich), 0.002\%

$\mathrm{v} / \mathrm{v} \quad$ 4-Dodecylbenzenesulfonic acid (Sigma-Aldrich), and $1 \% \quad \mathrm{v} / \mathrm{v} \quad$ (3- 
Glycidyloxypropyl)trimethyoxysilane (Sigma-Aldrich) was mixed together in a glass vial. The resultant solution was bath sonicated (Ultrasonic Cleaner, VWR) for 30 minutes and flowed through a $0.45 \mu \mathrm{m}$ filter (EZFlow Syringe Filter, Thomas Scientific) prior to use.

\subsubsection{Surface Preparation}

Microscope coverslips $(25 \times 25 \mathrm{~mm}$ No.1.5, VWR $)$ were cleaned with $45 \mathrm{~mL} 50 \%$ hydrogen peroxide (Sigma-Aldrich) and $105 \mathrm{~mL}$ 95-98\% sulfuric acid (VWR) for 10 minutes and then rinsed under $18.2 \mathrm{M} \Omega$-cm water for 30 minutes. Right before using, slides were washed again with $18.2 \mathrm{M} \Omega-\mathrm{cm}$ water and dried with an ultra-pure nitrogen stream. Prior to coating with PEDOT:PSS film, surfaces were treated with oxygen plasma (Harrick Plasma, Ithaca, NY). Microscope coverslips and Q-sensors (QSX $303 \mathrm{SiO}_{2}$, Biolin Scientific, Sweden) were treated with oxygen plasma under the maximum radio frequency power of $18 \mathrm{~W}$ for 2 minutes and 45 seconds, respectively. Glass or $\mathrm{SiO}_{2}$ surfaces were used immediately. To prepare PEDOT:PSS surfaces, glass or $\mathrm{SiO}_{2}$ supports were spin-coated (Apogee Spin coater, Cost Effective Equipment) with PEDOT:PSS at $2500 \mathrm{rpm}$ for 35 seconds,followed by annealing at $140{ }^{\circ} \mathrm{C}$ for 1 hour. Coated PEDOT:PSS surfaces were immersed into $18.2 \mathrm{M} \Omega$-cm water for 4 hours and dried with an ultrapure stream of nitrogen and treated with oxygen plasma for 2 minutes, just before use.

\subsubsection{Lipid Bilayer Formation}

A microfluidic chamber, which was provided by The Engineering in Translational Science Group at Nanyang Technical University, was stuck to the fresh cleaned glass or PEDOT:PSS surfaces. The microfluidic chamber consists of a straight channel device with two ports, an inlet and outlet, through which the solutions are fed and expelled.

To form bilayers using VF, liposome solution was delivered to the microfluidic channel, incubated for 30 minutes, then rinsed with PBS buffer to remove excess vesicles. 
To form a SALB, the lipid solution was delivered into the microfluidic channel and incubated for 30 minutes. Next, $10 \mathrm{mM}$ Tris buffer (pH7.5) with $150 \mathrm{mM} \mathrm{NaCl}$ at $50 \mu \mathrm{L} / \mathrm{min}$ was delivered to the channel for 60 minutes to gently remove excessive lipids in the bulk solution while forming the supported bilayer on the channel surface.

\subsubsection{Fluorescence Recovery after Photobleaching (FRAP) on Bilayers}

After bilayers are formed on glass or PEDOT:PSS surfaces, FRAP was carried out to confirm bilayer formations and determine the fluidity of the SLBs. With all lipid compositions, 0.5\% (mol/mol) Texas Red $\quad$ 1,2-Dihexadecanoyl-sn-Glycero-3-Phosphoethanolamine, Triethylammonium Salt (TR-DHPE) from Thermo Fisher was doped into the formulations as a fluorescent probe. The experimental setup consists of an inverted Zeiss Axio Observer.Z1 microscope with $\alpha$ Plan-Apochromat $20 \times$ objective. A $20 \mu \mathrm{m}$ diameter spot at the z-plane of the bilayer was bleached by $150 \mathrm{~mW} 561 \mathrm{~nm}$ optically pumped semiconductor laser (Coherent, Inc.) for $500 \mathrm{~ms}$. The recovery of the bleached spot was monitored for 30 minutes and the fluorescence intensity of the spot was measured and normalized to a reference spot in each image. The normalized fluorescence intensity against time was fit with a Bessel function following the method of Soumpasis et al. ${ }^{67}$ and then the following equation, $D=w^{2} / 4 t_{1 / 2}$ where $\mathrm{w}$ is the radius of the photobleached spot and $t_{1 / 2}$ is the time required to achieve half of the maximum recovery intensity. From this expression, the diffusion coefficient as calculated.

\subsubsection{Quartz Crystal Microbalance with Dissipation (QCM-D) Monitoring Lipid Bilayer}

\section{Formation}

The Q-sense E1 (Biolin Scientific, Sweden) instrument was used to monitored the lipid bilayer formation process in real time during VF or SA methods. The changes of frequency and energy dissipation were collected at the $1^{\text {st }}, 3^{\text {rd }}, 5^{\text {th }}, 7^{\text {th }}, 9^{\text {th }}, 11^{\text {th }}, 13^{\text {th }}$ overtones; data reported in 
this paper were measured at the third overtone. All the experiments were executed at a temperature setting of $25.0 \pm 0.5{ }^{\circ} \mathrm{C}$ under a steady flow rate of $50 \mu \mathrm{L} / \mathrm{min}$, which was controlled by a peristaltic pump (ISMATEC). Before the experiment, organic contamination on the surface was removed by using oxygen plasma, as described above. Q-sensors (QSX $303 \mathrm{SiO}_{2}$, Biolin Scientific, Sweden) were prepared as described above. For PEDOT:PSS coated QCM crystals, sensors were initially stabilized by continuously flowing in buffer for 2.5 hours, and re-equilibrated to obtain a baseline prior to starting the bilayer formation experiments.

For VF experiments, PBS buffer was delivered and allowed to stabilize for 12 minutes. Next, liposome solution was delivered for 30 minutes. Lastly, PBS buffer was used to rinse out the chamber and excess bulk material.

For SALB experiments, Tris/ $\mathrm{NaCl}$ buffer was delivered to the chamber for 12 minutes followed by the isopropanol and water solvent $(3: 7 \mathrm{v} / \mathrm{v})$ with a flow rate of $50 \mu \mathrm{L} / \mathrm{min}$ for another 10 minutes. Once stabilized, the lipid mixture was continuously delivered at the same flow rate for 30 minutes. In the final step, solvent was exchanged at $50 \mu \mathrm{L} / \mathrm{min}$ by delivering Tris/ $\mathrm{NaCl}$ buffer to the chamber for 15 minutes.

\subsubsection{Quartz Crystal Microbalance with Dissipation (QCM-D) Monitoring the Insertion of}

\section{Alpha-Hemolysin and Disruption of Polymyxin B}

For mammalian model membrane, $\alpha$-hemolysin $(\alpha-H L)$ from staphylococcus aureus (Sigma-Aldrich) lyophilized powder was dissolved in $10 \mathrm{mM}$ Tris buffer (pH7.5) with $150 \mathrm{mM}$ $\mathrm{NaCl}$ at $0.5 \mathrm{mg} / \mathrm{mL}$, and then diluted to a $5 \times 10^{-6} \mathrm{mg} / \mathrm{mL}$ concentration immediately before using. For bacterial model membrane, polymyxin B (PMB) sulfate salt (Sigma-Aldrich) powder was dissolved in Tris/ $\mathrm{NaCl}$ buffer at $0.5 \mathrm{mg} / \mathrm{mL}$ right before using. After the SALB formed on the PEDOT:PSS film, and the signal stabilized, $\alpha$-hemolysin or polymyxin B were delivered to the 
mammalian model membrane or bacterial model membrane under the flow rate of $50 \mu \mathrm{L} / \mathrm{min}$ for 15 minutes, respectively. Then, the Tris/ $\mathrm{NaCl}$ was delivered to the chamber to rinse out excess material for 15 minutes. The same procedures were used for the EIS measurements in this work.

\subsubsection{Electrochemical Impedance Spectroscopy Measurement for Lipid bilayer formation} and Detection of Membrane Interactions

Impedance spectra of the PEDOT:PSS/SLB platform were measured using an Autolab potentiostat equipped with a frequency response analysis module. Commercially available $\mathrm{Ag} / \mathrm{AgCl}$ electrode and a platinum mesh $(45 \mathrm{~mm} \times 25 \mathrm{~mm})$ were used as the reference and counter electrodes, respectively. The working PEDOT:PSS electrode had a round shape and a diameter of $300 \mu \mathrm{m}$. The counter electrode was sufficiently large in order to have minimal contribution on the impedance values measured. The applied AC voltage was $0.01 \mathrm{~V}$ and a DC voltage of $0 \mathrm{mV}$ versus open-circuit potential. The electrolyte solution was PBS, unless otherwise stated. The measured impedance spectra were fitted to a model represented by equivalent circuits as shown in Figure $\mathrm{S} 2$ in the supplementary information using the EC-lab software. For the bilayers, the equivalent circuit consists of an electrolyte resistance and two RC elements (a resistance connected in parallel to a capacitance) in series.

\subsection{Results and discussion}

\subsubsection{Supported Lipid Bilayer Formation by Vesicle Fusion or Solvent-Assisted Methods}

The vesicle fusion (VF) method has been used as a standard process to fabricate cell membrane mimics on solid supports. It proceeds by vesicle adsorption on hydrophilic surfaces, such as glass, driven by van der Waals forces that also drives the vesicle rupture into bilayer patches. ${ }^{68}$ At a critical coverage of vesicles, a contiguous lipid bilayer is formed as the individual 
bilayer patches merge together when their edges touch ${ }^{69}$ For this method to be successful, several conditions must be met. There must be sufficient attraction between the vesicle and the surface, which constrains both the composition and surface properties that will promote VF. Second, the surface must be flat so that topographical features do not impede the rupture and/or edge healing process. Given these constraints, there are a rather limited set of surfaces and vesicle compositions that can be used. Thus, to form bilayers on other surface types, i.e., polymers, alternative assembly processes are often necessary.

In this study, a solvent-assisted (SA) method ${ }^{60}$ was used to assemble SLBs on glass and PEDOT:PSS surfaces. SA has the merits of minimal preparation time and is less influenced by the supporting surface material. In this method, lipids undergo phase transitions in the solvent; initially, micelles are suspended in the isopropanol/water mixture. Lipid micelles adsorb to the surface during incubation. Then, as the solvent is slowly exchanged with an increasing water fraction, micelles rapidly self-assemble into bilayer patches that interconnect to form a contiguous lipid bilayer (Fig. 1A).

\subsubsection{Characterization of Lipid Bilayer Planarity and Two-dimensional Fluidity on Glass and PEDOT:PSS Surfaces}

After lipid bilayer formation was attempted by either VF or SA methods on glass or PEDOT:PSS surfaces, their planarity and two-dimensional fluidity (mobility) was determined by FRAP. To carry out these experiments, an approximately $20 \mu \mathrm{m}$ diameter circle was bleached in the fluorescently labeled bilayers using a laser beam. An intensity profile was obtained by drawing a line across the photobleached region at each time point; and then the data was normalized to the background intensity of 100 to compare the data across different samples. From the recovery of the fluorescent intensity of this circle over time, the two-dimensional lipid diffusion coefficient in 
the bilayer and its mobile fraction was obtained. Additionally, the recovery of fluorescence at the focused z-plane of the bilayer confirmed the formation of a contiguous, planar lipid bilayer over micron length scale of the photobleached region.

In the first set of experiments, POPC and cholesterol (4:1) were formulated as a commonly used model of the mammalian membrane and bilayers made using the SA method (Fig. 2 A-B). Interestingly, cholesterol-rich domains were observed in the lipid bilayer for glass substrates (Fig. 2A), which has been previously reported for this composition by the SA method on glass by Tabaei et al. ${ }^{36}$

To compare the fluidity of the lipid bilayers prepared by the SA method against conventional VF, a second set of FRAP experiments were carried out on bilayers made by VF with the same composition. Figure $\mathbf{2 C}$ shows the recovery of the mammalian model membrane made by VF on glass. As expected, the supported lipid bilayer readily formed on the glass and exhibited typical diffusion coefficients and mobile fractions ${ }^{36}$ However, VF of this composition did not proceed on the PEDOT:PSS surface, as indicated by the lack of recovery of the photobleached spot (Fig. 2D). PEDOT:PSS is a negatively charged, conducting polymer and the swelling behavior of the polymer film in the aqueous buffer increases the surface roughness, thus the vesicles must overcome a higher energy barrier for adsorption and rupture to form a planar lipid bilayer on this surface. ${ }^{21,31}$ 

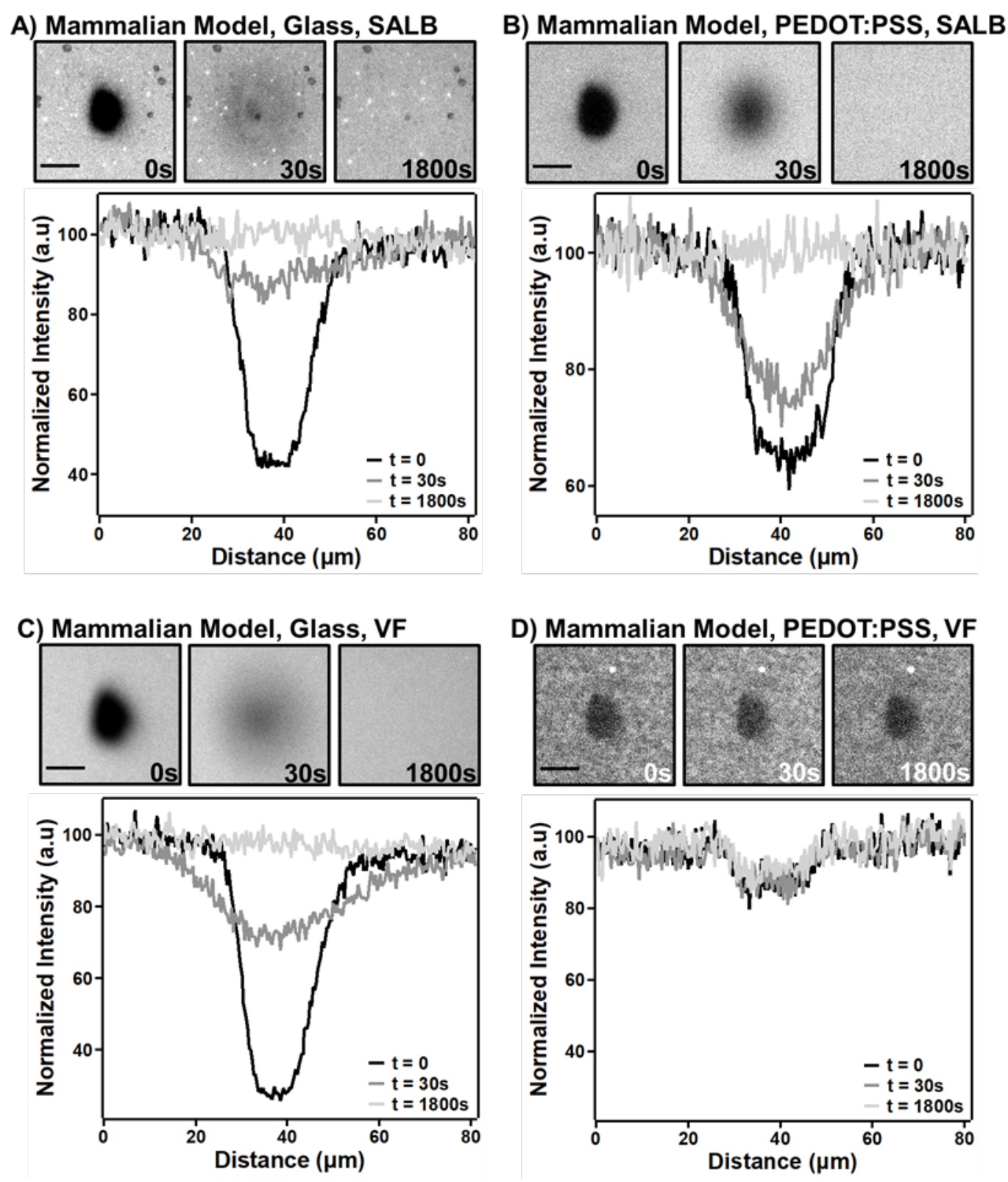

Figure 2. FRAP results of mammalian model membrane on glass and PEDOT:PSS surfaces. The scale bar in all images is $20 \mu \mathrm{m}$. The plots show representative line scans across the center of the image and normalized as described in the text to compare across samples. (A) SALB on the glass substrate (B) SALB on the PEDOT:PSS film (C) VF lipid bilayer on the glass substrate (D) Unsuccessful VF (intact vesicles) on the PEDOT:PSS film.

The quantitative summary of SA and VF lipid bilayers fluidity on the glass and PEDOT:PSS surface are listed in Table 1. For mammalian membranes on glass, the diffusion coefficient of the lipid bilayer made by SA was comparable with the lipid bilayer made by VF, moreover; the mobile fraction of the SA lipid bilayer was similar to the VF lipid bilayer. The 
variance of the diffusivity and mobile fractions for fluid lipid bilayers on glass made by SA or VF fell within the same range. More importantly, for PEDOT:PSS surfaces, we found that the only way to form this typical supported bilayer formulation was by the SA method. The SA method not only facilitated the lipid bilayer formation on the negatively charged polymer surface, but achieved stable and reproducible qualities. However, while the mobile fraction is high for all samples, indicating that bilayer quality is very good, we note that the diffusion coefficient is smaller on the PEDOT:PSS surface. Due to the high mobile fraction, reduced diffusivity cannot be due to the presence of more immobile lipids, so this points to either a change in the bilayer itself or that there may be more surface area within the bleached area on the polymer compared to glass.

\begin{tabular}{|c|c|c|c|}
\hline \multicolumn{4}{|c|}{ Table 1. Diffusivity and Mobile Fraction of Mammalian Membranes on Glass and } \\
PEDOT:PSS using SA and VF \\
\hline Surface & Method & Diffusivity $\left(\boldsymbol{\mu \mathbf { m } ^ { 2 } / \mathbf { s } )}\right.$ & Mobile Fraction \\
\hline Glass & SA & $1.46 \pm 0.36$ & $0.93 \pm 0.03$ \\
\hline PEDOT:PSS & SA & $0.23 \pm 0.04$ & $0.95 \pm 0.05$ \\
\hline Glass & VF & $1.09 \pm 0.21$ & $0.99 \pm 0.01$ \\
\hline PEDOT:PSS & VF & NA & NA \\
\hline
\end{tabular}

To emulate a bacterial membrane, POPE and POPG (4:1) was used as a model composition. FRAP experiments were used to characterize the lipid bilayer formation, diffusion, and mobility on the glass and PEDOT:PSS substrates made by the SA method (Fig. 3A-B). The recovery of the photobleached spot confirms the formation of this bacteria model membrane on both glass and PEDOT:PSS surfaces. Critically, for both glass and PEDOT:PSS surfaces, this formulation of vesicle does not rupture to form a planar bilayer by VF (Fig. 3C-D). The reasons for this failure are two-fold. The intrinsic negative curvature of the POPE lipids, which have conically spatial structure, are not prone to the deformation that promotes vesicle rupture. More significant is the inclusion of negatively charged lipids, POPG, a defining feature of bacteria membranes, that 
creates an electrostatic repulsion between these vesicles and the negatively charged glass or PEDOT:PSS surfaces.
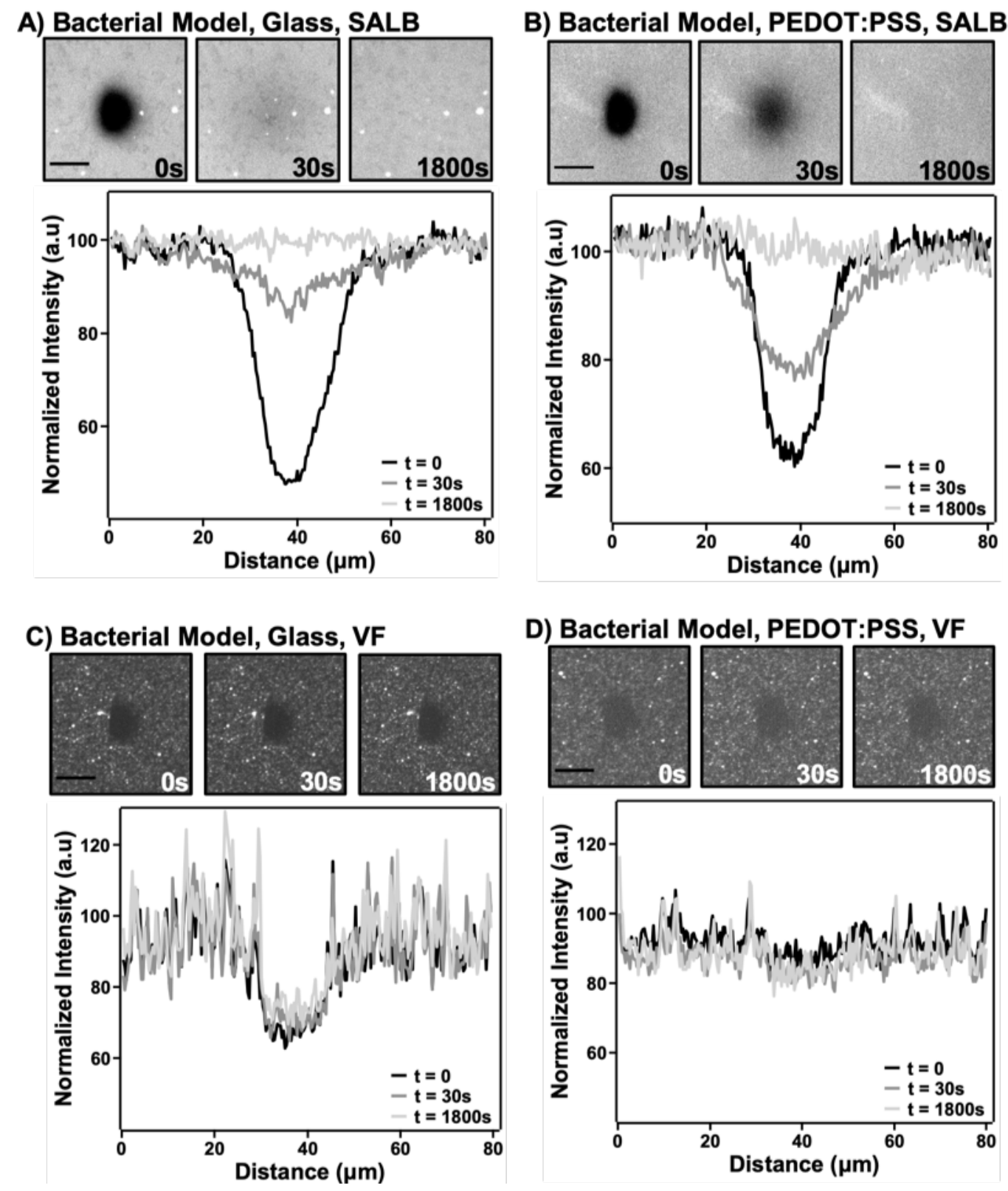

Figure 3. FRAP results of bacterial model membrane on the glass and PEDOT:PSS substrates. The normalized intensity was obtained by drawing a line across the center of the photobleached area. Scale bar is $20 \mu \mathrm{m}$ (A) the fluorescent image and normalized intensity of SALB on the glass substrate (B) the fluorescent image and normalized intensity of SALB on the PEDOT:PSS film. (C) The fluorescent image and normalized intensity of intact vesicles on the glass substrate. (D) The fluorescent image and normalized intensity of intact vesicles on the PEDOT:PSS film. 
Table 2 summarizes the results for bacterial model membrane mobility characteristics. The results for diffusion coefficient and mobile fraction indicate that the SA method can form fluidized lipid bilayers by SA method but not by VF. We observe again that the SA method on both glass and PEDOT:PSS have high mobile fractions, but the diffusion coefficient on the CP is lower, as observed in the mammalian case. Since both of these very different bilayers are behaving with the same trend, we conclude that the reduction in diffusivity is most likely due to an increased surface area on the swollen CP compared to the rigid, flat glass surface.

Since both of these very different bilayers are behaving with the same diffusion trend, on PEDOT:PSS there are several factors that might affect the diffusivity overall on this surface. First, an increased surface area on the swollen CP compared to the rigid glass surface would give rise to a larger amount of the material in the photobleached spot, which can result in calculation of a lower diffusion coefficient during recovery if not strictly accounted for. Another, perhaps more likely factor, is the change in the hydrophilicity of the polymer layer and the possibility for lipids to momentarily pin on the polymer. The addition of the co-solvent additive EG and crosslinker GOPS can affect the hydrophilicity of the PEDOT:PSS. ${ }^{21,70}$ To be specific, with the EG content increase from 0 to $20 \%$, previous studies have shown by water contact angle measurement that the hydrophilicity decreases after soaking in the DI water. ${ }^{21}$ In addition to EG in our formulation, the crosslinker GOPS is another compound that can affect (decrease) the hydrophilicity, as shown by previous work. ${ }^{70}$ These factors, however, can be counteracted by the $\mathrm{O}_{2}$ plasma treatment by introducing hydroxyl groups at the surface, which give rise to a more hydrophilic character. ${ }^{21}$ Therefore, with the typically used PEDOT:PSS formulation (5\% v/v/ EG, 1\% v/v GOPS, 0.002\% $\mathrm{v} / \mathrm{v}$ DBSA), post $\mathrm{O}_{2}$ plasma treatment, the hydrophilicity is adequate to promote SALB formation on the surface. Nonetheless, these chemical changes may affect the diffusion coefficient due to 
strong lipid interactions with the polymer itself that can lead to pinning. Lastly, surface roughness can affect the diffusion coefficient. It has been documented that greater roughness of glass surfaces results in lower diffusion coefficients in supported bilayers. ${ }^{71}$ The inherent roughness of the glass slides coated in polymer used here gives rise to an even rougher surface ${ }^{21,71}$. This roughness can result in small defects in the lipid bilayer that can lead to reduce diffusion coefficients when measured using FRAP. ${ }^{72}$

Table 2. Diffusivity and Mobile Fraction of Bacterial Membrane on Glass and PEDOT:PSS using SA and VF

\begin{tabular}{|c|c|c|c|}
\hline Surface & Method & Diffusivity $\left(\boldsymbol{\mu \mathbf { m } ^ { 2 } / \mathbf { s } )}\right.$ & Mobile Fraction \\
\hline Glass & SA & $1.28 \pm 0.49$ & $1.06 \pm 0.05$ \\
\hline PEDOT:PSS & SA & $0.30 \pm 0.05$ & $0.94 \pm 0.06$ \\
\hline Glass & VF & NA & NA \\
\hline PEDOT:PSS & VF & NA & NA \\
\hline
\end{tabular}

\subsubsection{Monitoring of the Real-Time Lipid Bilayer Formation on $\mathrm{SiO}_{2}$ and PEDOT:PSS}

\section{Films by SA Method}

In this work, QCM-D was used to monitor the lipid bilayer formation in the real-time, by measuring the change of the resonance frequency $(\Delta f)$ and energy dissipation $(\Delta D)$, parameters that structure of absorbed films. The $\Delta f$ is related to the amount of lipid mass adsorbed on the surface, and the $\Delta D$ is related to the viscoelastic properties of the adsorbed layer.

For SALB experiments, after obtaining the baseline for PEDOT:PSS stabilized in $\mathrm{NaCl} /$ Tris buffer at $50 \mu \mathrm{l} / \mathrm{min}$ flowrate, isopropanol and water solvent was delivered into the chamber and a commensurate decrease in frequency was observed from the change in viscosity of the solution. After the signal stabilized, the lipid mixture in the identical solvent was delivered into the chamber. As the lipids deposited on the surface, a further decrease of the frequency was 
observed. Finally, lipid bilayer was formed during the solvent exchange process with the initial buffer.

Figure 4A-B shows the mammalian bilayer formation process on the $\mathrm{SiO}_{2}$ sensor and PEDOT:PSS film. For the lipid bilayer on the $\mathrm{SiO}_{2}$ surface, the values of $\Delta f$ and $\Delta D$ confirmed the formation of supported lipid bilayers on the QCM-D crystal, which are summarized in Table 3. Those values are in alignment with similar compositions in the literature made by either SA or VF methods. ${ }^{36}$ For lipid bilayers on the PEDOT:PSS, the $\Delta f$ resulted in larger differences than on the bare crystals. This result points to a larger amount of adsorbed material on the PEDOT:PSS that may occur due to a larger surface area after polymer swelling in the aqueous buffer or individual lipids or micelles which may have permeated into the PEDOT:PSS films. Moreover, the $\Delta D$ is larger for mammalian-mimetic bilayers on PEDOT:PSS films compared to the values on the bare sensors. One hypothesis is that as PEDOT:PSS is mechanically softer than glass, so the energy dissipation is also higher. ${ }^{22}$ However, more likely is that there are either individual lipids or micelles that did not incorporate into the bilayer, and either cause a nanoscale defect at that location, or if they penetrate into the polymer network, this may lead to a higher $\Delta D$ as well. ${ }^{73} \mathrm{We}$ will return to this point later, as this is not observed in the bacteria mimetic bilayer case. QCM-D also confirms here that the VF method does not form planar bilayers on PEDOT:PSS (see data in Supplementary Information); corresponding frequency and dissipation values are listed in Table 3 for comparison and show much higher mass and dissipation .

Figure 4C-D shows the bacterial bilayer formation process on the $\mathrm{SiO}_{2}$ sensor and PEDOT:PSS film, with the final values in Table 3. The key points to note here are: 1) VF does not work on either glass or PEDOT:PSS surfaces. 2) The bacteria-mimetic SALBs are consistent in $\Delta f$ and $\Delta D$ values between glass and PEDOT:PSS, which are also well aligned with the values 
of the mammalian-mimetic SALB on glass. 3) Comparing the mammalian and bacterial SALBs on PEDOT:PSS, the mammalian bilayer leads to larger mass uptake and more changes in dissipation. This is consistent with the idea that the zwitterionic nature of the mammalian composition allows some free lipids and micelles to partition into the PEDOT:PSS during solvent phase. We note that the bacterial SALB does not show this behavior because it consists of primarily negatively charged species and thus has less propensity to interact with the negatively charged PEDOT:PSS surface. This is also reflected in the comparison of the VF data between the mammalian and bacterial vesicles: the mammalian case has a significantly higher mass adsorbed and dissipation than the bacterial case, indicating the stronger affinity of the mammalian vesicles for PEDOT:PSS compared to the bacterial vesicles. Our previous work has shown that these vesicles are too big to enter the PEDOT:PSS network, so these values reflect the difference in surface adsorption and affinity for the PEDOT:PSS. ${ }^{50}$ As a final point, we note that one way to limit the adsorption into the PEDOT:PSS could be to minimize the length of time the surface is exposed to the lipid/solvent mixture but we have not examined that parameter here. 
A) Mammalian Model, $\mathrm{SiO}_{2}$, SALB
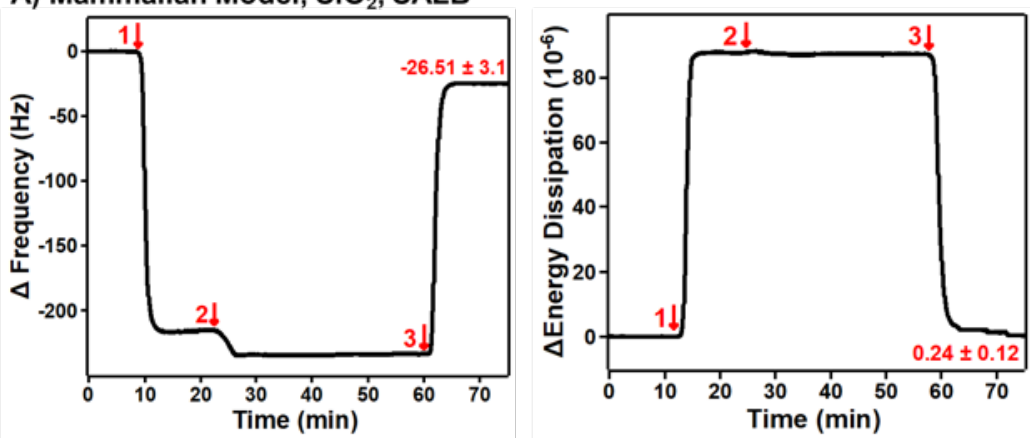

B) Mammalian Model, PEDOT:PSS, SALB
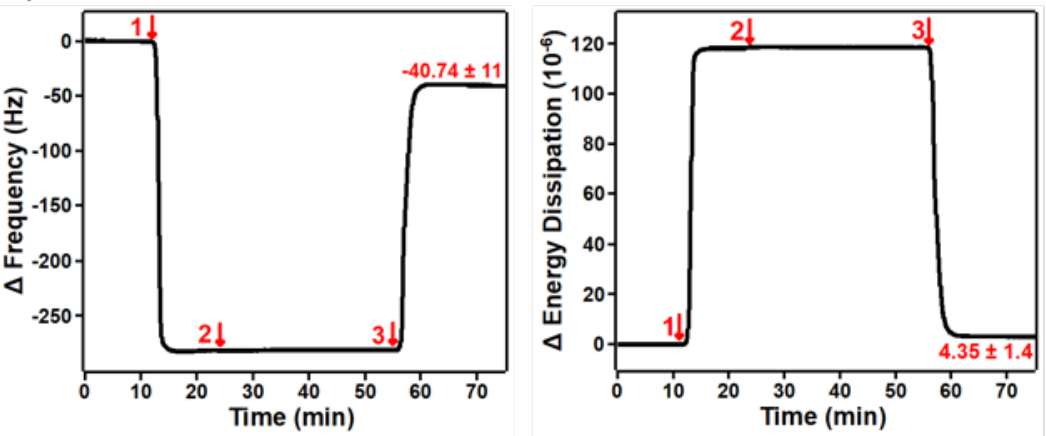

C) Bacterial Model, $\mathrm{SiO}_{2}$, SALB
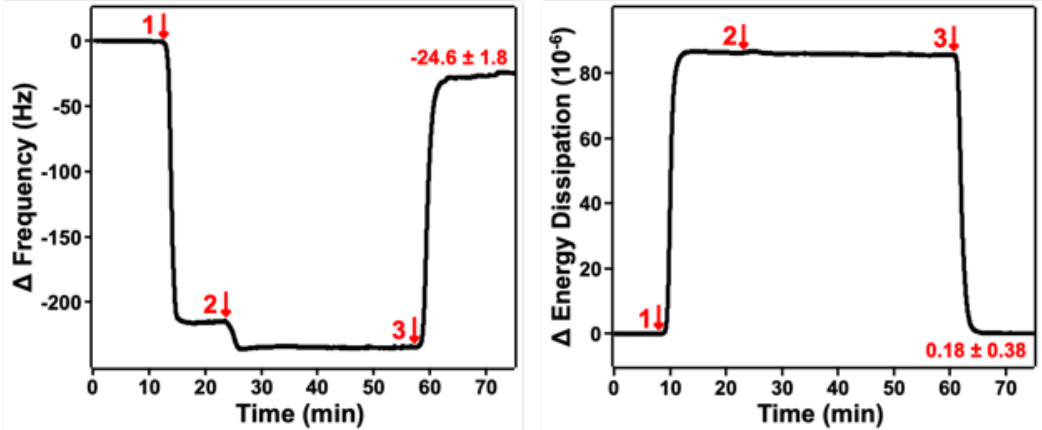

D) Bacterial Model, PEDOT:PSS, SALB
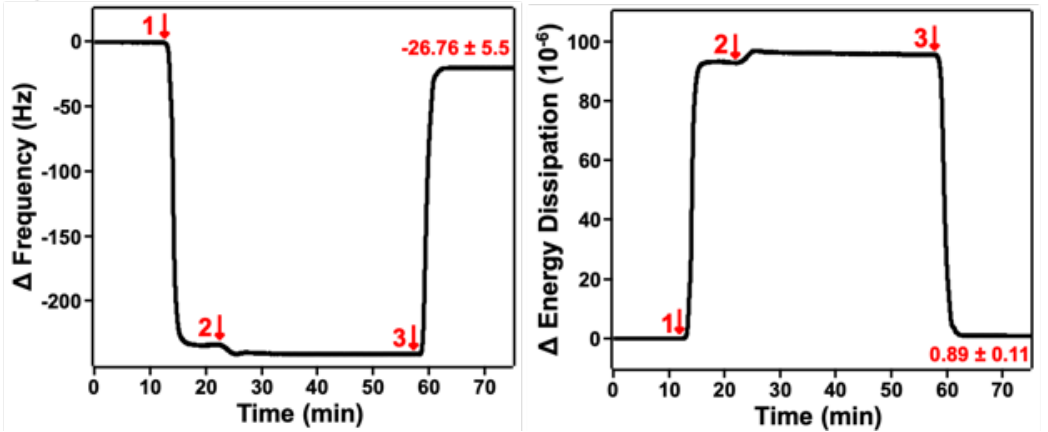

Figure 4. QCM-D monitoring the SALB on glass and PEDOT:PSS surfaces. (A) Mammalian model membrane on the sensor $\left(\mathrm{SiO}_{2}\right)$. (B) Mammalian model membrane on the PEDOT:PSS coated sensor. (C) Bacterial model membrane on the sensor $\left(\mathrm{SiO}_{2}\right)$. (D) Bacterial model membrane on the PEDOT:PSS coated sensor. The measurements of frequency $(\Delta f)$ and energy dissipation $(\Delta D)$ were shown under the third overtone. The error bars were calculated by the means of the results for at least three experiments. The arrows indicate time of delivery of: (1) the water and isopropanol solvent to the sensor, (2) the lipid mixture in the identical solvent, and (3) solvent exchange with Tria/ $\mathrm{NaCl}$ buffer to the chamber. 


\begin{tabular}{|c|c|c|c|c|}
\hline $\begin{array}{c}\text { Biomembrane } \\
\text { Model }\end{array}$ & Substrates & $\begin{array}{c}\text { Bilayer } \\
\text { formation } \\
\text { method }\end{array}$ & $\begin{array}{c}\text { Frequency } \\
(\Delta \mathbf{f})\end{array}$ & $\begin{array}{c}\text { Energy } \\
\text { Dissipation }(\Delta D, \\
10-6)\end{array}$ \\
\hline Mammalian & $\mathrm{SiO}_{2}$ & SA & $-26.5 \pm 3.1$ & $0.24 \pm 0.12$ \\
\hline Mammalian & $\mathrm{SiO}_{2}$ & VF & $-31.23 \pm 1.4$ & $0.46 \pm 0.19$ \\
\hline Mammalian & PEDOT:PSS & SA & $-40.7 \pm 11$ & $4.35 \pm 1.4$ \\
\hline Mammalian & PEDOT:PSS & VF & $-140.9 \pm 37$ & $15.64 \pm 3.1$ \\
\hline Bacterial & $\mathrm{SiO}_{2}$ & SA & $-24.6 \pm 1.8$ & $0.18 \pm 0.38$ \\
\hline Bacterial & $\mathrm{SiO}_{2}$ & VF & $-10.21 \pm 3.8$ & $1.29 \pm 0.41$ \\
\hline Bacterial & PEDOT:PSS & SA & $-26.8 \pm 0.05$ & $0.89 \pm 0.11$ \\
\hline Bacterial & PEDOT:PSS & VF & $-2.26 \pm 1.51$ & $0.55 \pm 0.50$ \\
\hline
\end{tabular}

After lipid bilayers formed on the PEDOT:PSS coated QCM-D sensor, we tested the ability to insert $\alpha$-HL into the mammalian model membrane and disrupt the bacterial model with the antibiotic compound, Polymyxin B (PMB). Figure 5 A shows the QCM-D signal during the $\alpha-H L$ insertion into the mammalian model membrane. The large frequency decrease after delivering $\alpha$ HL to the chamber can be attributed to the additional mass detected on the bilayer during the protein's interaction with membrane surface. There may be insertion to form a pore, but it is not possible to assess the nature of this interaction from the dissipation, which does not change significantly. It could be naively assumed that there should be a shift to a larger dissipation due to the water replacing the membrane inside the pores, however, it may be more complicated than that. There are several possible reasons no change is observed: 1) the method is not sensitive enough to measure the small change in dissipation at this concentration of protein; 2) the part of the membrane that is now replaced by the protein and water is not more dissipative that the lipids 
themselves. This could be true if there was significant hydrogen bonding in the ion channel and if the protein itself was "rigid", further, when the protein inserts it compressed the lipid tails elsewhere to make room and thus could lead to a slight increase in tail ordering that could sum to a slightly stiffer and less dissipative bilayer overall. The third possibility is that no protein insertion occurred. However, the EIS data (presented in the next section) tells us that at least some protein must have inserted to explain the change in resistance. Thus, we cautiously conclude that the QCM-D results corroborate a favorable interaction of the protein with the bilayer and the EIS results tell us that at least some of those proteins are inserted.

Figure 5 B shows the PMB disruption of bacterial model membrane by QCM-D. In this case we see that the frequency increases, meaning that membrane material is lost from the surface, presumably due to the disruption (soluabilization) by PMB of the bacterial membrane. Again, the dissipation signal is easy to over interpret because no significant change is detected, but it seems reasonable that if the membrane was removed in large part from the surface, the dissipation should fall close to the value before the bilayer was formed (plain PEODT:PSS surface). This value is already very small, even with the intact bilayer on it, so it may just not be possible with the resolution of QCM-D to see this change in dissipation. However, with the EIS results, we can clearly see a change in resistance only when the PMB is present, so that supports that the PMB is causing this change in bilayer integrity. 
A) Mammalian Model with $\alpha-H L$, PEDOT:PSS, SALB
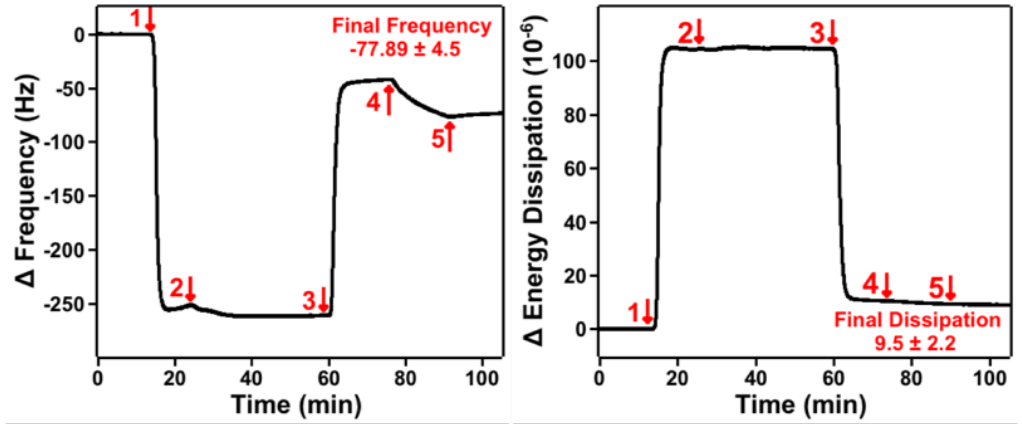

B) Bacterial Model with PMB, PEDOT:PSS, SALB
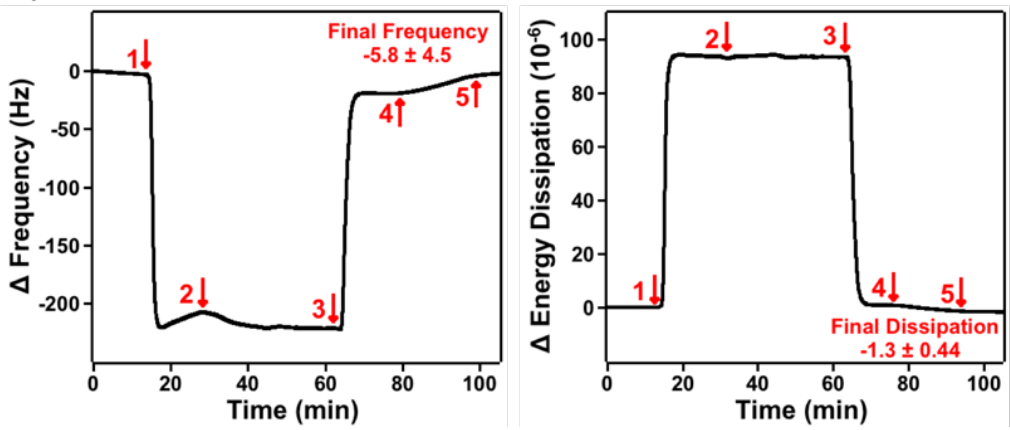

Figure 5. QCM-D monitoring the SALB interaction with $\alpha$-HL or PMB on PEDOT:PSS films. (A) Mammalian model membrane interacts with $\alpha-H L$. (B) Bacterial model membrane interacts with PMB. The measurements of frequency $(\Delta f)$ and energy dissipation $(\Delta D)$ were shown under the third overtone. The results are the means \pm s.d. from three independent experiments. The arrows indicate time of delivery of: (1) the water and isopropanol solvent to the sensor, (2) the lipid mixture in the identical solvent, and (3) solvent exchange with Tris/ $\mathrm{NaCl}$ buffer to the chamber. (4) $\alpha$ HL and PMB deliver to the mammalian model and bacterial model, respectively. (5) rinse excessive material with Tris/ $\mathrm{NaCl}$ buffer to the chamber.

\subsubsection{Characterization of Electrical Properties of Lipid Bilayers on PEDOT:PSS Electrode}

\section{Surfaces}

The electrochemical impedance spectra of the PEDOT:PSS electrodes before and after formation of the SLB were recorded to characterize the change in electrical properties of the electrode. Figure 6 presents the phase of the impedance and the Nyquist plot representation of the complex impedance data before and after formation of the SLB on a PEDOT:PSS electrode. When the SLB assembles, we observe a shift in the phase plots for both the mammalian and the bacterial membranes, which is a consequence of an additional layer forming on top of PEDOT:PSS. The lipid layer modulates the ionic current flux sensed by the electrode. Formation of the bilayer 
generates the characteristic semi-circle in the Nyquist plot, indicative of an RC element (Fig. 6B, D). We model the EIS data with an equivalent electrical circuit (Fig. S2) commonly used for the interpretation of the complex impedance of SLBs and obtain the corresponding resistance and capacitance values for each bilayer. ${ }^{70}$ Due to the inherent inhomogeneity and porosity of PEDOT:PSS, the constant phase element (CPE) is used in the equivalent electrical circuit instead of an ideal capacitor (C), to take into consideration the unknown aspects of the electrode surface and the nature of the interfacial layer interacting with the lipid bilayer. ${ }^{70,74-76}$ The model consists of serially connected impedance elements representing: i) the ohmic behavior of the buffer $\left(R_{1}\right.$, which also includes other uncompensated resistances associated with leads and contacts), ii) the impedance of the lipid bilayer $\left(\mathrm{R}_{2}\right.$ in parallel with $\left.\mathrm{CPE}_{2}\right)$, and iii) the impedance element consisting of a resistor $\left(\mathrm{R}_{3}\right)$ and the $\mathrm{CPE}_{3}$ to account for the PEDOT:PSS film. ${ }^{77}$ The calculated resistance of the SLBs was found to be $0.54 \pm 0.05 \mathrm{k} \Omega * \mathrm{~cm}^{2}$ and $0.46 \pm 0.09 \mathrm{k} \Omega * \mathrm{~cm}^{2}$ for the mammalian and the bacterial SLB, respectively. The capacitance was calculated to be $376.6 \pm 166.1 \mu \mathrm{F} / \mathrm{cm}^{2}$ and $950.0 \pm 808.7 \mu \mathrm{F} / \mathrm{cm}^{2}$ for the mammalian and the bacterial SLB, respectively. We attribute the higher capacitance values of the bacterial membrane model to the electrostatic interactions between the negatively charged PSS regions on top of the CP surface with the negatively charged lipid domains of our bacterial membrane, inducing an additional membrane-solution interface acting as an ionic reservoir. We note that our mammalian model membrane resistance is one magnitude higher than previous work reports of membrane resistance of several $\Omega \mathrm{cm}^{2} .^{70}$ This comparison indicates that the lipid bilayers made by the SA method exhibit better membrane sealing on the conducting polymer surface compared to those formed from similar compositions via vesicle fusion. 
The typical compositions used to study bacterial membranes in literature are POPC/POPG or POPC/POPE/POPG. Though PC lipids are not found in bacterial membranes, they are often included in model membrane studies, due to the difficulties using formulations with POPE and POPG alone. A POPC/POPE/POPG (4:3:1) mixture prepared by evaporating solvent and rehydrating the lipid thin film on an ITO surface results in a membrane with resistance of $4.2 \mathrm{k} \Omega \mathrm{cm}^{2}{ }^{78}$ In this work, we are able to remove the irrelevant POPC component and demonstrate the formation of the POPE/POPG lipid bilayer by using the SA method on PEDOT:PSS. The resistance of this membrane is $0.46 \pm 0.09 \mathrm{k} \Omega * \mathrm{~cm}^{2}$, however the capacitance is higher from typically reported ones made by other methods ${ }^{79,80}$

The overall higher capacitance values of our models compared to typical SLBs reported in literature could be attributed to the unknown and probably complex interface between the CP PEDOT:PSS and the membrane. Elucidating this requires further investigation using ultra sensitive methods (i.e., neutron reflectometry). This may lead to useful information regarding the effect of hydrated polymers on SLB formation and properties, possibly leading to more advanced equivalent circuit models that take into account the specific attributes of such interfaces.

In the next set of experiments, we investigated the effect of bacterial toxin insertion on the electrical properties of the mammalian SLB. As a toxin, we chose alpha-hemolysin ( $\alpha-\mathrm{HL})$, a transmembrane protein from Staphylococcus aureus which inserts into lipid bilayers to form pores.$^{81}$ As seen in Fig. 6A-B, the addition of $\alpha$-HL causes a decrease in the resistance of the SLB: change in resistance $(\Delta \mathrm{R})$ is $23.2 \% \pm 17.8 \%$ ( $\mathrm{R}$ value drops to $0.41 \pm 0.10 \mathrm{k} \Omega * \mathrm{~cm}^{2}$ from $0.54 \pm$ $0.05 \mathrm{k} \Omega^{*} \mathrm{~cm}^{2}$ ). We interpret this change as an indication of the interactions of the toxin with the bilayer, increasing its leakiness thus resulting in higher ion flux across it. For the bacterial membrane model, Polymyxin B (PMB), a commonly used antibacterial compound with a detergent 
like mechanism of action was chosen to test the disruption capability. ${ }^{82-84}$ In this case, as seen in Fig. 6C-D, we observe that the impedance spectra, both the phase and Nyquist plot, resemble the lipid bilayer-free (PEDOT:PSS only) state, indicating a possible destruction of the lipid bilayer with PMB ${ }^{84} \mathrm{PMB}$ causes a significant drop in the resistance of the membrane, i.e., $\Delta \mathrm{R}=99.63 \%$ $\pm 1.86 \%$ ( $\mathrm{R}$ value drops to $7.42 \pm 10.76 \Omega * \mathrm{~cm}^{2}$ from $0.46 \pm 0.09 \mathrm{k} \Omega * \mathrm{~cm}^{2}$ ). The change in bilayer resistance is a useful parameter to evaluate the integrity of the membrane. Here our work demonstrates EIS detection of antimicrobial compound interaction with a complete bilayer structure, which is more representative of the complete membrane. This platform therefore proves itself as a versatile tool to study antibacterial compound mechanism and potency via electrical monitoring. 
A
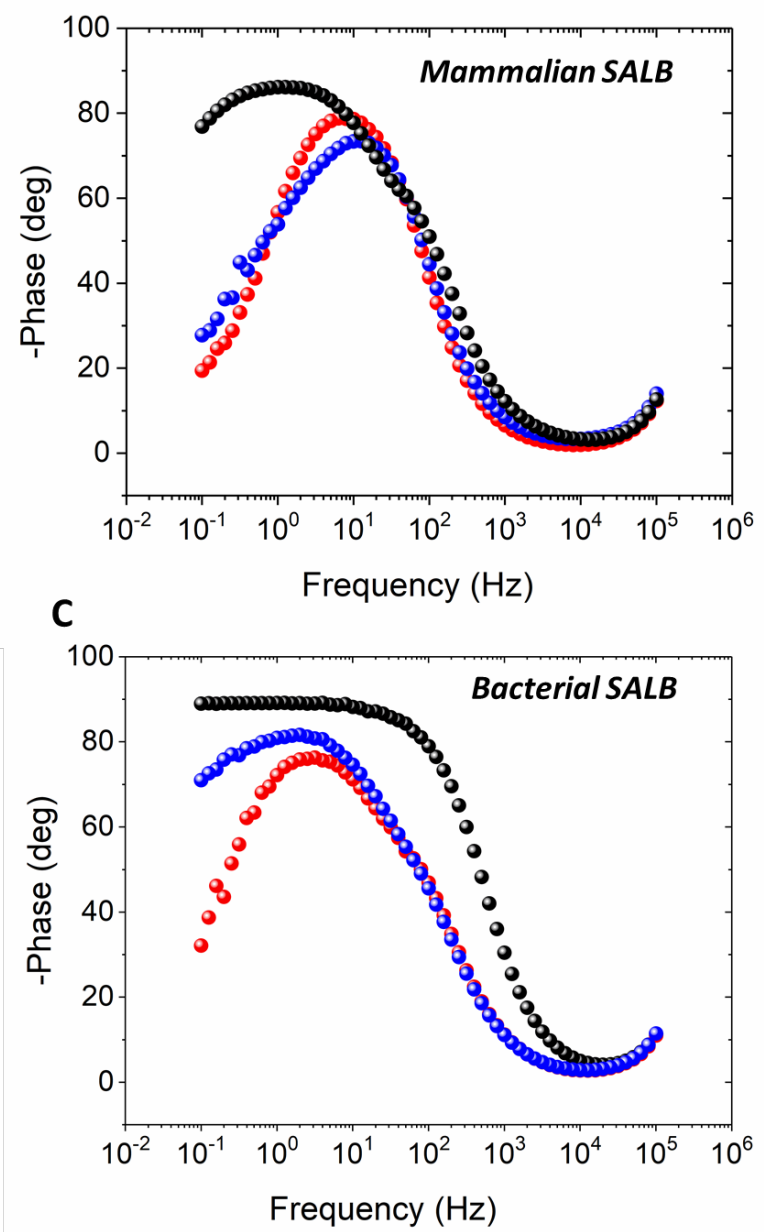

B

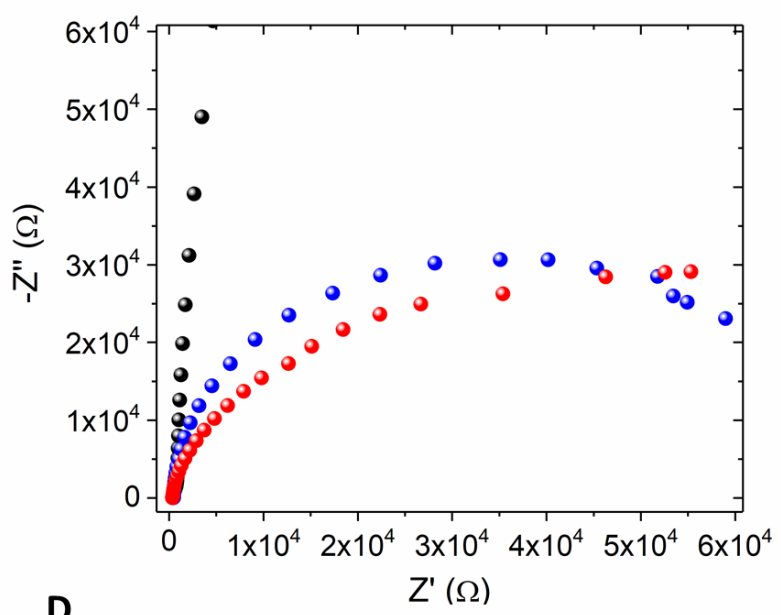

D

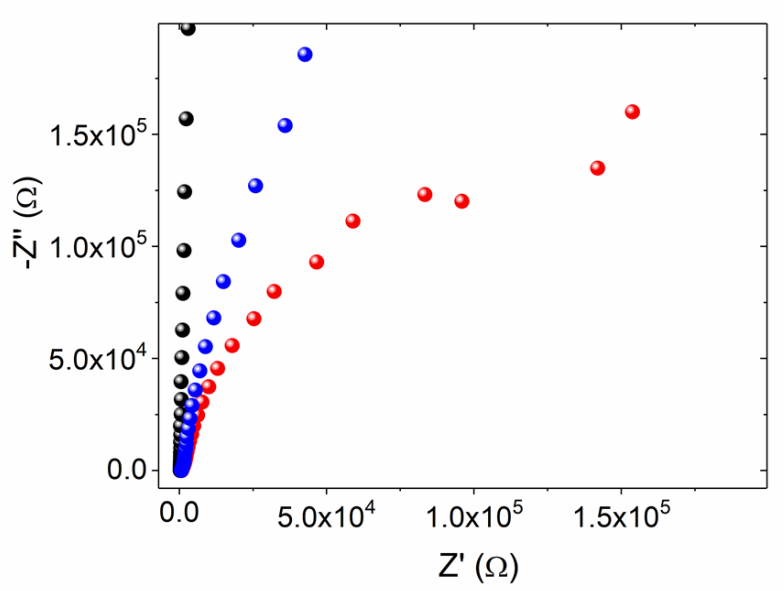

Figure 6. Impedance monitoring of the SALBs on Au/PEDOT:PSS electrodes. Phase of the impedance and the corresponding Nyquist plot for the (A, B) Mammalian membrane model and (C, D) Bacterial membrane model. Black symbols: before the SALB addition; Red symbols: after the SALB addition; and Blue symbols: after the insertion of the toxin $\alpha-\mathrm{HL}\left(5 \times 10^{-6} \mathrm{M}\right)$ or PMB $(0.5 \mathrm{mg} / \mathrm{ml})$ in the mammalian and in the bacterial bilayers, respectively.

\subsection{Conclusions}

The SALB method enables lipid bilayer formation on conducting polymer surfaces for compositions that are not possible with vesicle fusion. This critical advance opens this SLB platform for enhanced biosensing applications. With this electronic SLB platform, a means for label-free assessment of complex interactions are possible between mammalian membranes and bacterial toxins and bacteria membranes with antibiotic compounds. Building from this work, it 
will be possible in the future to conduct more advanced SLB studies using conducting polymer surfaces in more sophisticated organic bioelectronic devices, such as transistors, which may further expand our ability to sense small events at membrane surfaces. Furthermore, the compatibility with microfluidic devices enables a direct path towards massive parallelization of devices. Such future development will speed up antibiotic screening and novel drug discovery specific to gramnegative bacteria without specificity to mammalian cells that promotes preparedness for combatting antibiotic resistant strains.

\subsection{Acknowledgements}

We thank Professor Nam-Joon Cho (The Engineering in Translational Science Group at Nanyang Technical University) for his advice and providing the microfluidic flow cells used in this work. P. C would like to acknowledge funding from the Fundação de Amparo à Pesquisa do Estado de São Paulo (project 2018/14801-9). Research was sponsored by the Defense Advanced Research Projects Agency (DARPA) Army Research Office and was accomplished under Cooperative Agreement Number W911NF-18-2-0152. The views and conclusions contained in this document are those of the authors and should not be interpreted as representing the official policies, either expressed or implied, of DARPA or the Army Research Office or the U.S. Government. The U.S. Government is authorized to reproduce and distribute reprints for Government purposes notwithstanding any copyright notation herein. 


\section{CHAPTER 3 \\ FUTURE DIRECTION}

\subsection{SALB is the Platform to Study Variety of Biomembrane Activities}

Cell membrane is a fascinating structure where biological events happen, such as protein lipid interaction, membrane fusion, and toxin receptor interaction. Nowadays, it is appealing to use bioelectronics and electrochemical methods to monitor membrane activities. Therefore, it is critical to employ a conducting polymer as the interface between the biological system and electronics. SALB is the versatile platform which can be easily performed and fabricated on the PEDOT:PSS film, which is biocompatible and highly sensitive for membrane biosensing.

\subsubsection{Investigation of the Lipid and Solvent Interaction with PEDOT:PSS Film}

From this work, it has proven that SALB method is a simple and efficient approach to form lipid bilayers on the PEDOT:PSS film. However, the diffusion coefficient of the lipid bilayer formed on the PEDOT:PSS film is lower than the lipid bilayers formed on the glass, which may attribute to the lipid and PEDOT:PSS film interaction. PEDOT:PSS used in the experiment is a acidic solution with a $\mathrm{pH}$ value of $1.5-2.5$, thus cause lipid hydroxyl groups protonated, which may alter the lipid interaction with the PEDOT:PSS film. To investigate the effect of $\mathrm{pH}$, guanidine can be added to adjust the $\mathrm{pH}$ of PEDOT:PSS solution, and then fluorescence recovery after photobleaching technique will be used to measure the diffusivity of lipid bilayers. Solvent and salt buffer may also impact the diffusivity since the ions could incorporate with the PEDOT:PSS film. Therefore, the X-ray photoelectron spectroscopy can be used to investigate whether the ions penetrate the PEDOT:PSS film. 


\subsubsection{The Interaction Between Lipid Bilayer and Bacterial Toxin and Antibacterial Drug}

In the QCM-D results, after the bacterial toxin and antibiotic deliver to the sensor, the shift of the frequency and energy dissipation indicate interactions between the lipid bilayers and the bacterial toxin and antibacterial drug. However, further characterizations are needed to investigate the exact mechanism of the interactions. The concentrations of the bacterial toxin and antibacterial drug will be adjusted. Serial dilution of the bacterial toxin and antibacterial drug will be performed to interrupt the lipid bilayers to understand the mechanism of the membrane insertion and disruption. QCM-D will be used to monitoring the frequency and energy dissipation change, and later analyzing the trend of the signal change. In addition to the QCM-D monitoring, EIS measurement will elucidate the membrane disrupting with the toxin or drug in real-time by monitor the electrochemical signal. 


\section{REFERENCE}

1. Singer, S. J.; Nicolson, G. L., The Fluid Mosaic Model of the Structure of Cell Membranes. Science 1972, 175 (4023), 720-731.

2. Qi, S. Y.; Groves, J. T.; Chakraborty, A. K., Synaptic pattern formation during cellular recognition. Proceedings of the National Academy of Sciences 2001, 98 (12), 6548.

3. Lee, A. G., How lipids affect the activities of integral membrane proteins. Biochimica et Biophysica Acta (BBA) - Biomembranes 2004, 1666 (1), 62-87.

4. Kholodenko, B. N., Cell-signalling dynamics in time and space. Nature Reviews Molecular Cell Biology 2006, 7 (3), 165-176.

5. Tamm, L. K.; McConnell, H. M., Supported phospholipid bilayers. Biophysical journal 1985, $47(1), 105-113$.

6. Hsu, H.-L.; Millet, J. K.; Costello, D. A.; Whittaker, G. R.; Daniel, S., Viral fusion efficacy of specific H3N2 influenza virus reassortant combinations at single-particle level. Scientific reports 2016, 6, 35537.

7. $\quad$ Tero, R.; Fukumoto, K.; Motegi, T.; Yoshida, M.; Niwano, M.; Hirano-Iwata, A., Formation of Cell Membrane Component Domains in Artificial Lipid Bilayer. Scientific Reports 2017, 7 (1), 17905.

8. Brown, J. S.; Mohamed, Z. J.; Artim, C. M.; Thornlow, D. N.; Hassler, J. F.; Rigoglioso, V. P.; Daniel, S.; Alabi, C. A., Antibacterial isoamphipathic oligomers highlight the importance of multimeric lipid aggregation for antibacterial potency. Communications Biology 2018, 1 (1), 220.

9. Blodgett, K. B., Films Built by Depositing Successive Monomolecular Layers on a Solid Surface. Journal of the American Chemical Society 1935, 57 (6), 1007-1022.

10. Ollivon, M.; Lesieur, S.; Grabielle-Madelmont, C.; Paternostre, M. t., Vesicle reconstitution from lipid-detergent mixed micelles. Biochimica et Biophysica Acta (BBA) Biomembranes 2000, 1508 (1), 34-50.

11. Lind, T. K.; Cárdenas, M.; Wacklin, H. P., Formation of Supported Lipid Bilayers by Vesicle Fusion: Effect of Deposition Temperature. Langmuir 2014, 30 (25), 7259-7263.

12. Liu, H.-Y.; Grant, H.; Hsu, H.-L.; Sorkin, R.; Bošković, F.; Wuite, G.; Daniel, S., Supported Planar Mammalian Membranes as Models of in Vivo Cell Surface Architectures. ACS Applied Materials \& Interfaces 2017, 9 (41), 35526-35538.

13. Richter, R. P.; Bérat, R.; Brisson, A. R., Formation of Solid-Supported Lipid Bilayers: An Integrated View. Langmuir 2006, 22 (8), 3497-3505.

14. Ye, Q.; Konradi, R.; Textor, M.; Reimhult, E., Liposomes Tethered to Omega-Functional PEG Brushes and Induced Formation of PEG Brush Supported Planar Lipid Bilayers. Langmuir 2009, 25 (23), 13534-13539.

15. Lind, T. K.; Wacklin, H.; Schiller, J.; Moulin, M.; Haertlein, M.; Pomorski, T. G.; Cárdenas, M., Formation and Characterization of Supported Lipid Bilayers Composed of Hydrogenated and Deuterated Escherichia coli Lipids. PLoS One 2015, 10 (12), e0144671e0144671.

16. Watts, T. H.; Gaub, H. E.; McConnell, H. M., T-cell-mediated association of peptide antigen and major histocompatibility complex protein detected by energy transfer in an evanescent wave-field. Nature 1986, 320 (6058), 179-181. 
17. Cho, N.-J.; Frank, C. W.; Kasemo, B.; Höök, F., Quartz crystal microbalance with dissipation monitoring of supported lipid bilayers on various substrates. Nature Protocols 2010, 5, 1096.

18. Quirós-Solano, W. F.; Gaio, N.; Silvestri, C.; Pandraud, G.; Sarro, P. M., PEDOT:PSS: A Conductive and Flexible Polymer for Sensor Integration in Organ-on-Chip Platforms. Procedia Engineering 2016, 168, 1184-1187.

19. Rivnay, J.; Inal, S.; Collins, B. A.; Sessolo, M.; Stavrinidou, E.; Strakosas, X.; Tassone, C.; Delongchamp, D. M.; Malliaras, G. G., Structural control of mixed ionic and electronic transport in conducting polymers. Nature Communications 2016, 7, 11287.

20. Rivnay, J.; Leleux, P.; Ferro, M.; Sessolo, M.; Williamson, A.; Koutsouras, D. A.; Khodagholy, D.; Ramuz, M.; Strakosas, X.; Owens, R. M.; Benar, C.; Badier, J.-M.; Bernard, C.; Malliaras, G. G., High-performance transistors for bioelectronics through tuning of channel thickness. Science Advances 2015, 1 (4), e1400251.

21. Zhang, Y.; Wustoni, S.; Savva, A.; Giovannitti, A.; McCulloch, I.; Inal, S., Lipid bilayer formation on organic electronic materials. Journal of Materials Chemistry C 2018, 6 (19), 52185227.

22. Rivnay, J.; Owens, R. M.; Malliaras, G. G., The Rise of Organic Bioelectronics. Chemistry of Materials 2014, 26 (1), 679-685.

23. Lang, U.; Müller, E.; Naujoks, N.; Dual, J., Microscopical Investigations of PEDOT:PSS Thin Films. Advanced Functional Materials 2009, 19 (8), 1215-1220.

24. Strakosas, X.; Bongo, M.; Owens, R. M., The organic electrochemical transistor for biological applications. Journal of Applied Polymer Science 2015, 132 (15).

25. Rivnay, J.; Inal, S.; Salleo, A.; Owens, R. M.; Berggren, M.; Malliaras, G. G., Organic electrochemical transistors. Nature Reviews Materials 2018, 3, 17086.

26. Håkansson, A.; Han, S.; Wang, S.; Lu, J.; Braun, S.; Fahlman, M.; Berggren, M.; Crispin, X.; Fabiano, S., Effect of (3-glycidyloxypropyl)trimethoxysilane (GOPS) on the electrical properties of PEDOT:PSS films. Journal of Polymer Science Part B: Polymer Physics 2017, 55 (10), 814-820.

27. Friedlein, J. T.; McLeod, R. R.; Rivnay, J., Device physics of organic electrochemical transistors. Organic Electronics 2018, 63, 398-414.

28. Pitsalidis, C.; Pappa, A. M.; Porel, M.; Artim, C. M.; Faria, G. C.; Duong, D. D.; Alabi, C. A.; Daniel, S.; Salleo, A.; Owens, R. M., Biomimetic Electronic Devices for Measuring Bacterial Membrane Disruption. Adv Mater 2018, 30 (39), e1803130.

29. Zhang, Y.; Inal, S.; Hsia, C. Y.; Ferro, M.; Ferro, M.; Daniel, S.; Owens, R. M., Supported Lipid Bilayer Assembly on PEDOT:PSS Films and Transistors. Advanced Functional Materials 2016, 26 (40), 7304-7313.

30. Puiggalí-Jou, A.; Pawlowski, J.; del Valle, L. J.; Michaux, C.; Perpète, E. A.; Sek, S.; Alemán, C., Properties of Omp2a-Based Supported Lipid Bilayers: Comparison with Polymeric Bioinspired Membranes. ACS Omega 2018, 3 (8), 9003-9019.

31. Duc, C.; Vlandas, A.; Malliaras, G. G.; Senez, V., Wettability of PEDOT:PSS films. Soft Matter 2016, 12 (23), 5146-5153.

32. Horii, T.; Hikawa, H.; Mochizuki, Y.; Okuzaki, H., Synthesis and Characterization of Highly Conductive PEDOT/PSS Colloidal Gels. 2012; Vol. 37, p 515-518.

33. Jackman, J. A.; Tabaei, S. R.; Zhao, Z.; Yorulmaz, S.; Cho, N.-J., Self-Assembly Formation of Lipid Bilayer Coatings on Bare Aluminum Oxide: Overcoming the Force of Interfacial Water. ACS Applied Materials \& Interfaces 2015, 7 (1), 959-968. 
34. Losada-Pérez, P.; Polat, O.; Parikh, A. N.; Seker, E.; Renner, F. U., Engineering the interface between lipid membranes and nanoporous gold: A study by quartz crystal microbalance with dissipation monitoring. Biointerphases 2018, 13 (1), 011002.

35. Tabaei, S. R.; Vafaei, S.; Cho, N.-J., Fabrication of charged membranes by the solventassisted lipid bilayer (SALB) formation method on $\mathrm{SiO} 2$ and $\mathrm{A} 12 \mathrm{O} 3$. Physical Chemistry Chemical Physics 2015, 17 (17), 11546-11552.

36. Tabaei, S. R.; Jackman, J. A.; Kim, S.-O.; Liedberg, B.; Knoll, W.; Parikh, A. N.; Cho, N.-J., Formation of Cholesterol-Rich Supported Membranes Using Solvent-Assisted Lipid SelfAssembly. Langmuir 2014, 30 (44), 13345-13352.

37. Wiegand, G.; Arribas-Layton, N.; Hillebrandt, H.; Sackmann, E.; Wagner, P., Electrical Properties of Supported Lipid Bilayer Membranes. The Journal of Physical Chemistry B 2002, 106 (16), 4245-4254.

38. Khan, M. S.; Dosoky, N. S.; Patel, D.; Weimer, J.; Williams, J. D., Lipid Bilayer Membrane in a Silicon Based Micron Sized Cavity Accessed by Atomic Force Microscopy and Electrochemical Impedance Spectroscopy. Biosensors (Basel) 2017, 7 (3), 26.

39. Atanasov, V.; Knorr, N.; Duran, R. S.; Ingebrandt, S.; Offenhäusser, A.; Knoll, W.; Köper, I., Membrane on a chip: a functional tethered lipid bilayer membrane on silicon oxide surfaces. Biophys J 2005, 89 (3), 1780-1788.

40. Briand, E.; Zäch, M.; Svedhem, S.; Kasemo, B.; Petronis, S., Combined QCM-D and EIS study of supported lipid bilayer formation and interaction with pore-forming peptides. Analyst 2010, 135 (2), 343-350.

41. Gamper, N.; Shapiro, M. S., Regulation of ion transport proteins by membrane phosphoinositides. Nature Reviews Neuroscience 2007, 8, 921.

42. Shi, Y.; Massagué, J., Mechanisms of TGF-\&\#x3b2; Signaling from Cell Membrane to the Nucleus. Cell 2003, 113 (6), 685-700.

43. Willumeit, R.; Kumpugdee, M.; Funari, S. S.; Lohner, K.; Navas, B. P.; Brandenburg, K.; Linser, S.; Andrä, J., Structural rearrangement of model membranes by the peptide antibiotic NK-2. Biochimica et Biophysica Acta (BBA)-Biomembranes 2005, 1669 (2), 125-134.

44. Syeda, R.; Holden, M. A.; Hwang, W. L.; Bayley, H., Screening blockers against a potassium channel with a droplet interface bilayer array. Journal of the American Chemical Society 2008, 130 (46), 15543-15548.

45. Cremer, P. S.; Boxer, S. G., Formation and spreading of lipid bilayers on planar glass supports. The Journal of Physical Chemistry B 1999, 103 (13), 2554-2559.

46. Cho, N.-J.; Frank, C. W.; Kasemo, B.; Höök, F., Quartz crystal microbalance with dissipation monitoring of supported lipid bilayers on various substrates. Nature protocols 2010, 5 (6), 1096.

47. Tanaka, M.; Sackmann, E., Polymer-supported membranes as models of the cell surface. Nature 2005, 437 (7059), 656-663.

48. Liu, H.-Y.; Chen, W.-L.; Ober, C. K.; Daniel, S., Biologically Complex Planar Cell Plasma Membranes Supported on Polyelectrolyte Cushions Enhance Transmembrane Protein Mobility and Retain Native Orientation. Langmuir 2018, 34 (3), 1061-1072.

49. Richards, M. J.; Hsia, C.-Y.; Singh, R. R.; Haider, H.; Kumpf, J.; Kawate, T.; Daniel, S., Membrane Protein Mobility and Orientation Preserved in Supported Bilayers Created Directly from Cell Plasma Membrane Blebs. Langmuir 2016, 32 (12), 2963-2974. 
50. Zhang, Y.; Inal, S.; Hsia, C. Y.; Ferro, M.; Ferro, M.; Daniel, S.; Owens, R. M., Supported lipid bilayer assembly on PEDOT: PSS films and transistors. Advanced Functional Materials 2016, 26 (40), 7304-7313.

51. Inal, S.; Hama, A.; Ferro, M.; Pitsalidis, C.; Oziat, J.; Iandolo, D.; Pappa, A.-M.; Hadida, M.; Huerta, M.; Marchat, D.; Mailley, P.; Owens, R. M., Conducting Polymer Scaffolds for Hosting and Monitoring 3D Cell Culture. Advanced Biosystems 2017, 1 (6), 1700052.

52. Braendlein, M.; Pappa, A.-M.; Ferro, M.; Lopresti, A.; Acquaviva, C.; Mamessier, E.; Malliaras, G. G.; Owens, R. M., Lactate Detection in Tumor Cell Cultures Using Organic Transistor Circuits. Advanced Materials 2017, 29 (13), 1605744.

53. Pappa, A.-M.; Curto, V. F.; Braendlein, M.; Strakosas, X.; Donahue, M. J.; Fiocchi, M.; Malliaras, G. G.; Owens, R. M., Organic Transistor Arrays Integrated with Finger-Powered Microfluidics for Multianalyte Saliva Testing. Advanced Healthcare Materials 2016, 5 (17), 22952302.

54. Khan, M. S.; Dosoky, N. S.; Berdiev, B. K.; Williams, J. D., Electrochemical impedance spectroscopy for black lipid membranes fused with channel protein supported on solid-state nanopore. European Biophysics Journal 2016, 45 (8), 843-852.

55. Van Gelder, P.; Dumas, F.; Winterhalter, M. J. B. c., Understanding the function of bacterial outer membrane channels by reconstitution into black lipid membranes. Biophysical Chemistry 2000, 85 (2-3), 153-167.

56. Horn, C.; Steinem, C., Photocurrents generated by bacteriorhodopsin adsorbed on nanoblack lipid membranes. Biophysical journal 2005, 89 (2), 1046-54.

57. Tabaei, S. R.; Choi, J.-H.; Haw Zan, G.; Zhdanov, V. P.; Cho, N.-J., Solvent-assisted lipid bilayer formation on silicon dioxide and gold. Langmuir 2014, 30 (34), 10363-10373.

58. Tabaei, S. R.; Vafaei, S.; Cho, N.-J., Fabrication of charged membranes by the solventassisted lipid bilayer (SALB) formation method on $\mathrm{SiO} 2$ and $\mathrm{Al} 2 \mathrm{O}$ 3. Physical Chemistry Chemical Physics 2015, 17 (17), 11546-11552.

59. Tabaei, S. R.; Ng, W. B.; Cho, S.-J.; Cho, N.-J., Controlling the formation of phospholipid monolayer, bilayer, and intact vesicle layer on graphene. ACS applied materials \& interfaces 2016, 8 (18), 11875-11880.

60. Ferhan, A. R.; Yoon, B. K.; Park, S.; Sut, T. N.; Chin, H.; Park, J. H.; Jackman, J. A.; Cho, N.-J., Solvent-assisted preparation of supported lipid bilayers. Nature Protocols 2019.

61. Hohner, A. O.; David, M. P. C.; Rädler, J. O., Controlled solvent-exchange deposition of phospholipid membranes onto solid surfaces. Biointerphases 2010, 5 (1), 1-8.

62. Reimhult, E.; Zäch, M.; Höök, F.; Kasemo, B., A Multitechnique Study of Liposome Adsorption on Au and Lipid Bilayer Formation on SiO2. Langmuir 2006, 22 (7), 3313-3319.

63. Richter, R.; Mukhopadhyay, A.; Brisson, A., Pathways of lipid vesicle deposition on solid surfaces: a combined QCM-D and AFM study. Biophysical journal 2003, 85 (5), 3035-47.

64. Merz, C.; Knoll, W.; Textor, M.; Reimhult, E., Formation of supported bacterial lipid membrane mimics. Biointerphases 2008, 3 (2), FA41.

65. Shatursky, O.; Heuck, A. P.; Shepard, L. A.; Rossjohn, J.; Parker, M. W.; Johnson, A. E.; Tweten, R. K., The mechanism of membrane insertion for a cholesterol-dependent cytolysin: a novel paradigm for pore-forming toxins. Cell 1999, 99 (3), 293-299.

66. Domingues, M. M.; Inácio, R. G.; Raimundo, J. M.; Martins, M.; Castanho, M. A.; Santos, N. C., Biophysical characterization of polymyxin B interaction with LPS aggregates and membrane model systems. Peptide Science 2012, 98 (4), 338-344. 
67. Soumpasis, D., Theoretical analysis of fluorescence photobleaching recovery experiments. Biophysical journal 1983, 41 (1), 95-97.

68. Hamai, C.; Yang, T.; Kataoka, S.; Cremer, P. S.; Musser, S. M., Effect of average phospholipid curvature on supported bilayer formation on glass by vesicle fusion. Biophysical journal 2006, 90 (4), 1241-8.

69. Mapar, M.; Joemetsa, S.; Pace, H.; Zhdanov, V. P.; Agnarsson, B.; Hook, F., Spatiotemporal Kinetics of Supported Lipid Bilayer Formation on Glass via Vesicle Adsorption and Rupture. J Phys Chem Lett 2018, 9 (17), 5143-5149.

70. Zhang, Y.; Inal, S.; Hsia, C. Y.; Ferro, M.; Ferro, M.; Daniel, S.; Owens, R. M. J. A. F. M., Supported lipid bilayer assembly on PEDOT: PSS films and transistors. 2016, 26 (40), 73047313.

71. Seu, K. J.; Pandey, A. P.; Haque, F.; Proctor, E. A.; Ribbe, A. E.; Hovis, J. S., Effect of Surface Treatment on Diffusion and Domain Formation in Supported Lipid Bilayers. Biophys $J$ 2007, 92 (7), 2445-2450.

72. Mulligan, K.; Jakubek, Z. J.; Johnston, L. J., Supported Lipid Bilayers on Biocompatible Polysaccharide Multilayers. Langmuir 2011, 27 (23), 14352-14359.

73. Tarabella, G.; Nanda, G.; Villani, M.; Coppedè, N.; Mosca, R.; Malliaras, G. G.; Santato, C.; Iannotta, S.; Cicoira, F., Organic electrochemical transistors monitoring micelle formation. Chemical Science 2012, 3 (12), 3432-3435.

74. Briand, E.; Zäch, M.; Svedhem, S.; Kasemo, B.; Petronis, S. J. A., Combined QCM-D and EIS study of supported lipid bilayer formation and interaction with pore-forming peptides. 2010, 135 (2), 343-350.

75. Chang, W. K.; Wimley, W. C.; Searson, P. C.; Hristova, K.; Merzlyakov, M. J. B. e. B. A.-B., Characterization of antimicrobial peptide activity by electrochemical impedance spectroscopy. 2008, 1778 (10), 2430-2436.

76. Paulsen, B. D.; Tybrandt, K.; Stavrinidou, E.; Rivnay, J. J. N. m., Organic mixed ionicelectronic conductors. 2019, 1-14.

77. Koutsouras, D. A.; Gkoupidenis, P.; Stolz, C.; Subramanian, V.; Malliaras, G. G.; Martin, D. C., Impedance Spectroscopy of Spin-Cast and Electrochemically Deposited PEDOT:PSS Films on Microfabricated Electrodes with Various Areas. ChemElectroChem 2017, 4 (9), 2321-2327.

78. Puiggalí-Jou, A.; Pawlowski, J.; del Valle, L. J.; Michaux, C.; Perpète, E. A.; Sek, S.; Alemán, C. J. A. o., Properties of Omp2a-based supported lipid bilayers: Comparison with polymeric bioinspired membranes. 2018, 3 (8), 9003-9019.

79. Wiedman, G.; Herman, K.; Searson, P.; Wimley, W. C.; Hristova, K., The electrical response of bilayers to the bee venom toxin melittin: evidence for transient bilayer permeabilization. Biochim Biophys Acta 2013, 1828 (5), 1357-1364.

80. Gross, L. C.; Heron, A. J.; Baca, S. C.; Wallace, M. I. J. L., Determining membrane capacitance by dynamic control of droplet interface bilayer area. 2011, 27 (23), 14335-14342.

81. Chalmeau, J.; Monina, N.; Shin, J.; Vieu, C.; Noireaux, V., $\alpha$-Hemolysin pore formation into a supported phospholipid bilayer using cell-free expression. Biochimica et Biophysica Acta (BBA) - Biomembranes 2011, 1808 (1), 271-278.

82. Pitsalidis, C.; Pappa, A.-M.; Porel, M.; Artim, C. M.; Faria, G. C.; Duong, D. D.; Alabi, C. A.; Daniel, S.; Salleo, A.; Owens, R. M., Biomimetic Electronic Devices for Measuring Bacterial Membrane Disruption. Advanced Materials 2018, 30 (39), 1803130. 
83. Tam, V.H.; Schilling, A. N.; Vo, G.; Kabbara, S.; Kwa, A. L.; Wiederhold, N. P.; Lewis, R. E., Pharmacodynamics of polymyxin B against Pseudomonas aeruginosa. Antimicrob Agents Chemother 2005, 49 (9), 3624-30.

84. Zavascki, A. P.; Goldani, L. Z.; Li, J.; Nation, R. L., Polymyxin B for the treatment of multidrug-resistant pathogens: a critical review. J Antimicrob Chemother 2007, 60 (6), 1206-15. 


\section{APPENDIX I: SUPPORTING MATERIAL FOR CHAPTER 2}

\section{S1. Vesicle Fusion on PEDOT:PSS Film or Bare Sensor Surface Measured by QCM-D}

For VF experiments, PEDOT:PSS films or bare sensor surfaces were first stabilized by flowing in PBS buffer with a flow rate of $50 \mu \mathrm{L} / \mathrm{min}$. Then, vesicles were delivered to the $\mathrm{SiO}_{2}$ sensor or PEDOT:PSS films and monitored for rupture. Finally, the excess vesicles were rinsed out of the chamber and the final equilibration reported the associated frequency and dissipation changes that reflect whether or not a bilayer formed. The only case here reflected the characteristic behavior and values indicative of bilayer formation from vesicle rupture is the mammalian model on the bare $\mathrm{SiO}_{2}$ sensor surface. These resultant values are in Table $\mathbf{3}$ in the main text. 
A) Mammalian Model, $\mathrm{SiO}_{2}$, VF
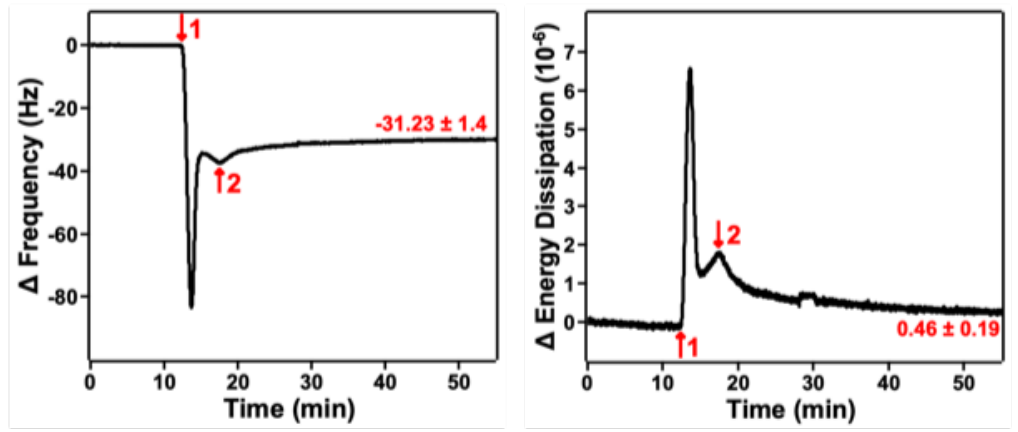

B) Mammalian Model, PEDOT:PSS, VF
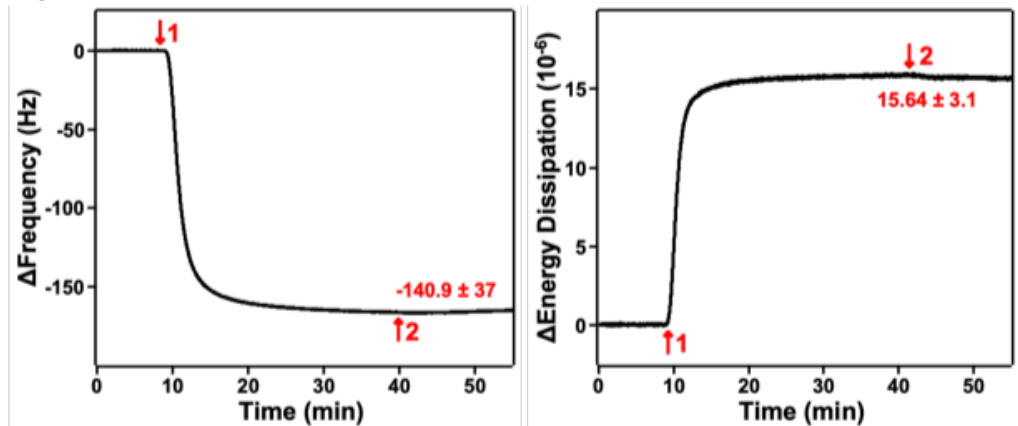

C) Bacterial Model, $\mathrm{SiO}_{2}$, VF
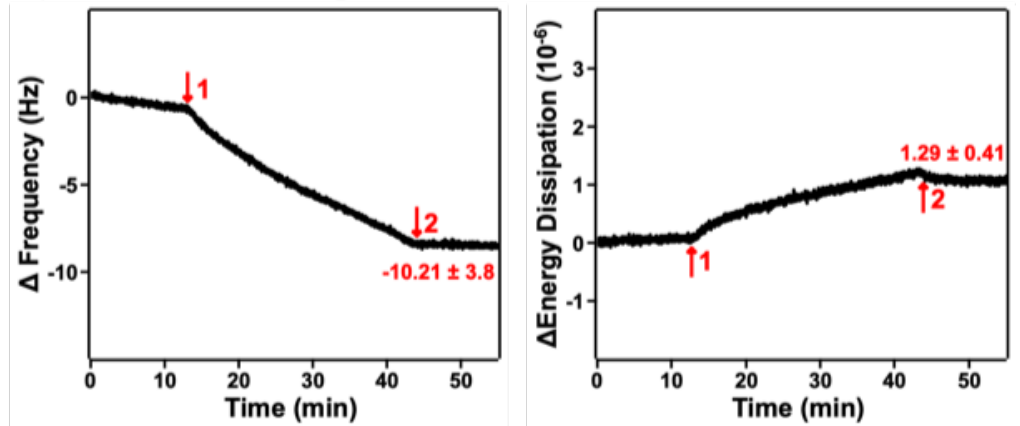

D) Bacterial Model, PEDOT:PSS, VF
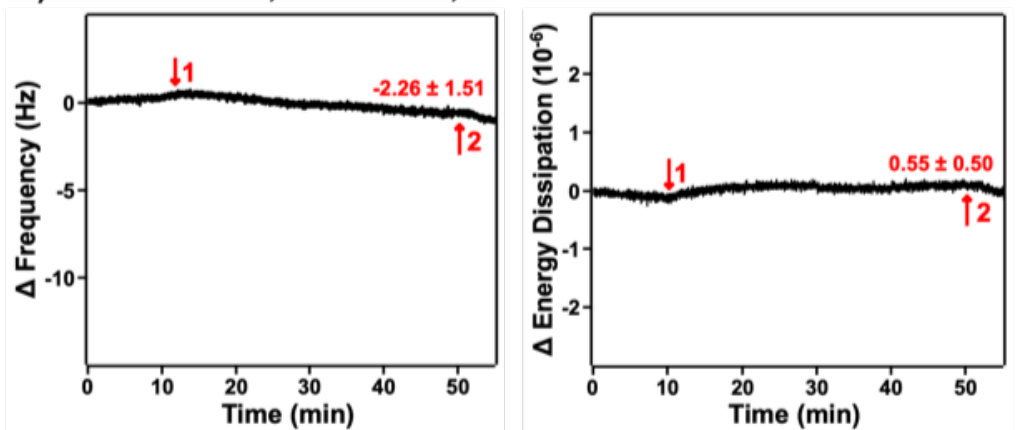

Figure S1. QCM-D monitoring the VF on glass and PEDOT:PSS surfaces. (A) Mammalian model membrane on the sensor $\left(\mathrm{SiO}_{2}\right)$. (B) Mammalian model membrane on the PEDOT:PSS coated sensor. (C) Bacterial model membrane on the sensor $\left(\mathrm{SiO}_{2}\right)$. (D) Bacterial model membrane on the PEDOT:PSS coated sensor. The measurements of frequency $(\Delta f)$ and energy dissipation $(\Delta D)$ were showed under the third overtone. The error bars were calculated by the means of the results for at least three experiments. The arrows indicate time of delivery: of (1) the lipid mixture to the sensor and (2) PBS rinsed excessive liposomes in the chamber. 


\section{S2. EIS Modelling of the Mammalian and the Bacterial Membranes}

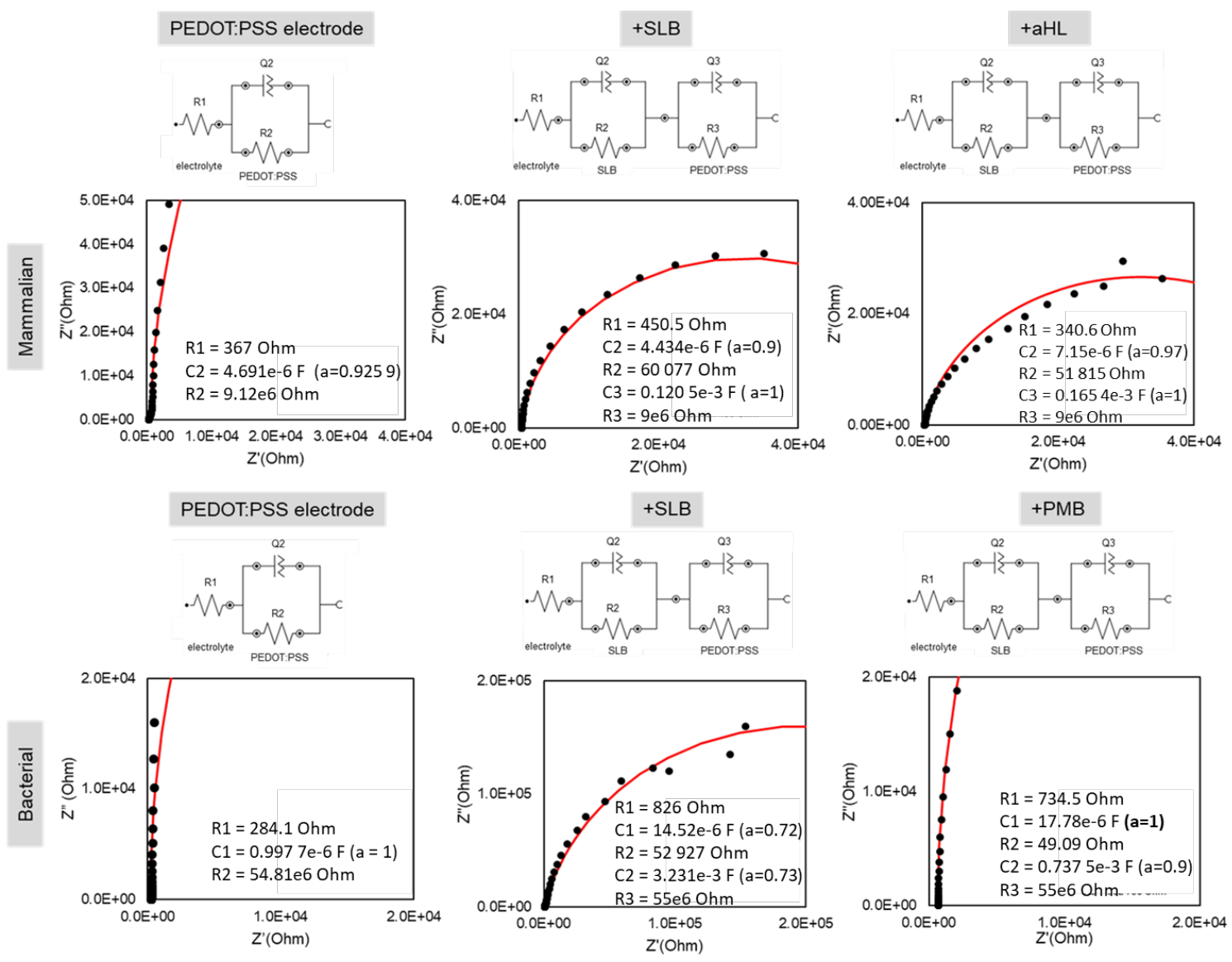

Figure S2. EIS modelling of the mammalian (top panel) and the bacterial (bottom panel) membranes on top of circular PEDOT:PSS electrodes of $0.0078 \mathrm{~cm}^{2}$. Red lines represent the fitted data and the black symbols the measured data. 


\section{S3. The Solvent Exchange Control Measured by QCM-D}

The QCM-D was used to prove the final shift of the frequency and energy dissipation were caused by the SALB formation rather than the shift of the solvent exchange in the chamber. The baseline was stabilized by delivering the Tris/NaCl buffer at a flow rate of $50 \mu \mathrm{L} / \mathrm{min}$. Next, isopropanol/water solvent was delivered to the QCM crystal, and then the solvent exchange by the Tris $/ \mathrm{NaCl}$ in the chamber. As shown in the figure below, solvent was continuously delivered to the sensor for 45 minutes. After solvent exchange by Tris/ $\mathrm{NaCl}$ in the chamber, the frequency and energy dissipation shift back to the baseline, indicating the final result of the $\Delta \mathrm{f}$ and $\Delta \mathrm{D}$ can only cause by the SALB formation rather than the solvent exchange.

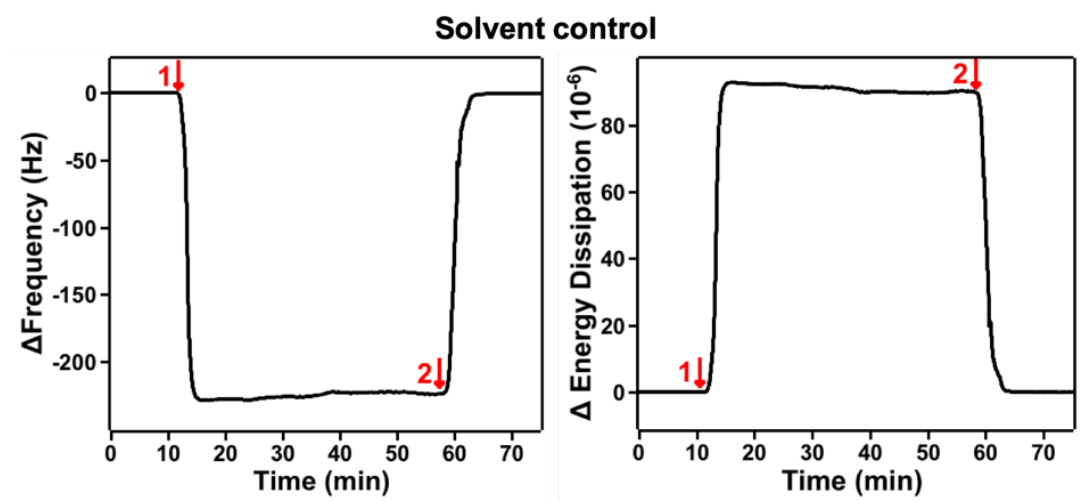

Figure S3.QCM-D monitoring the solvent-exchange process on the QCM crystal. The measurements of frequency $(\Delta \mathrm{f})$ and energy dissipation $(\Delta \mathrm{D})$ were shown under the third overtone. The initial baseline was obtained in the Tris/ $\mathrm{NaCl}$. The arrows indicate time of delivery of: (1) the water and isopropanol solvent to the sensor, (2) solvent exchange with the identical Tris/ $\mathrm{NaCl}$ buffer to the chamber. 
S4. The Insets of the Final Solvent Exchange of QCM-D Monitoring the SALB on Glass and PEDOT:PSS Surfaces

A) Mammalian Model, $\mathrm{SiO}_{2}$, SALB
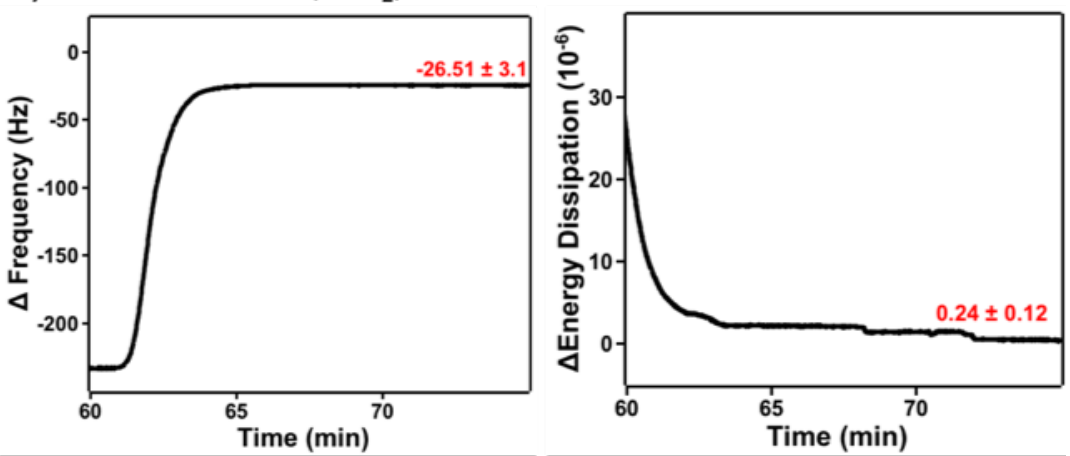

B) Mammalian Model, PEDOT:PSS, SALB
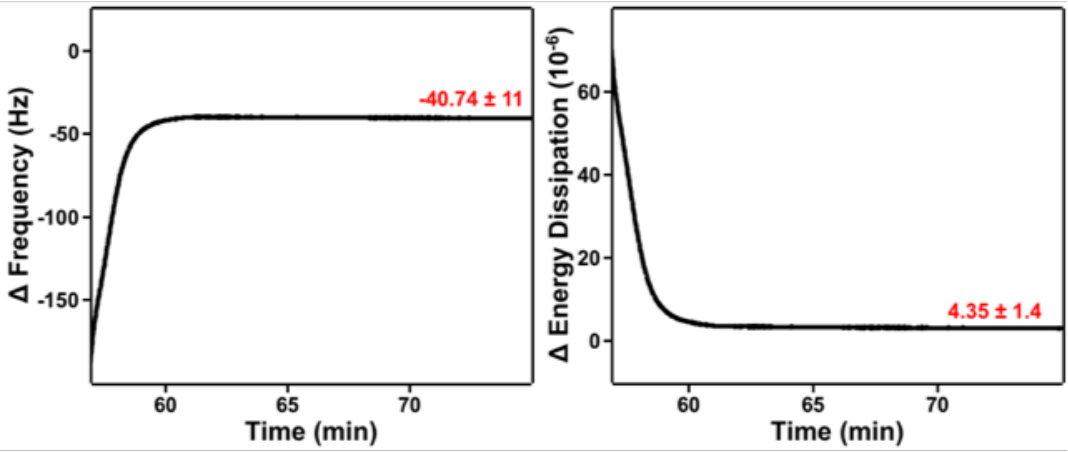

C) Bacterial Model, $\mathrm{SiO}_{2}, \mathrm{SALB}$
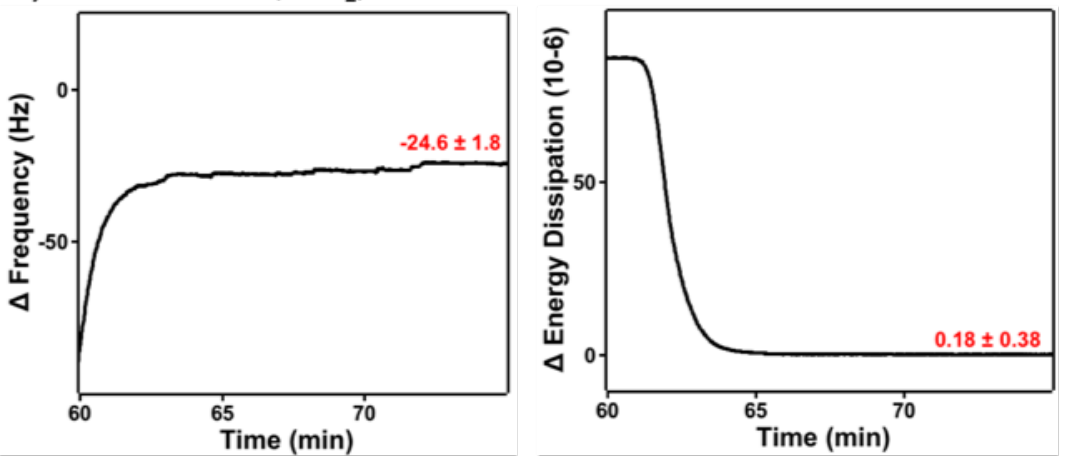

D) Bacterial Model, PEDOT:PSS, SALB
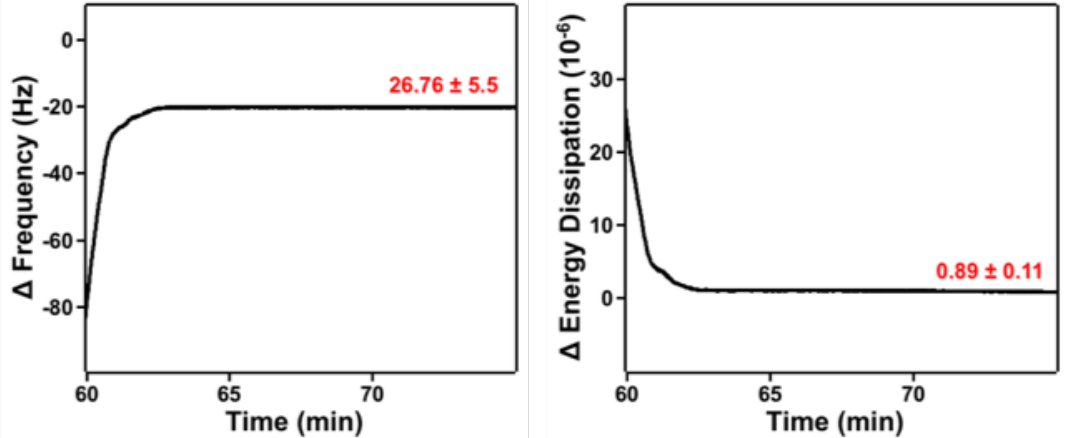
Figure S4. The insets of the final solvent exchange of QCM-D monitoring the SALB on glass and PEDOT:PSS surfaces. (A) Mamalian model membrane on the sensor $\left(\mathrm{SiO}_{2}\right)$. (B) Mammalian model membrane on the PEDOT:PSS coated sensor. (C) Bacterial model membrane on the sensor $\left(\mathrm{SiO}_{2}\right)$. (D) Bacterial model membrane on the PEDOT:PSS coated sensor. The measurements of frequency $(\Delta \mathrm{f})$ and energy dissipation $(\Delta \mathrm{D})$ were shown under the third overtone. The error bars were calculated by the means of the results for three experiments 